TRANSACTIONS OF THE

AMERICAN MATHEMATICAL SOCIETY

Volume 364, Number 4, April 2012, Pages 1933-1974

S 0002-9947(2011)05382-1

Article electronically published on November 17, 2011

\title{
EXISTENTIALLY CLOSED DIMENSION GROUPS
}

\author{
PHILIP SCOWCROFT
}

\begin{abstract}
A partially ordered Abelian group $\mathcal{M}$ is algebraically (existentially) closed in a class $\mathcal{C} \ni \mathcal{M}$ of such structures just in case any finite system of weak inequalities (and negations of weak inequalities), defined over $\mathcal{M}$, is solvable in $\mathcal{M}$ if solvable in some $\mathcal{N} \supseteq \mathcal{M}$ in $\mathcal{C}$. After characterizing existentially closed dimension groups this paper derives amalgamation properties for dimension groups, dimension groups with order unit, and simple dimension groups. By determining the quantifier-free types that may be isolated by existential formulas the paper produces many pairwise nonembeddable countable finitely generic dimension groups. The paper also finds several elementary properties distinguishing finitely generic dimension groups among existentially closed dimension groups. The paper finally embeds nontrivial dimension groups functorially into existentially closed dimension groups.
\end{abstract}

\section{INTRODUCTION}

A dimension group is a partially ordered Abelian group whose partial ordering is directed 11 and isolated $2^{2}$ and obeys the Riesz interpolation property 3 This paper will focus on dimension groups that are existentially closed (e.c.) when viewed as structures for the language $\mathcal{L}=\{+,-, 0, \leq\}$ of partially ordered Abelian groups. While a simple argument shows that a dimension group is algebraically closed (as an $\mathcal{L}$-structure) just in case it is divisible 4 , the nature of e.c. dimension groups is harder to determine. Section 2 exploits two ways of building new dimension groups from old to reveal six axioms, or axiom schemas, that are true in e.c. dimension groups. One of these schemas belongs to $\mathcal{L}_{\omega_{1} \omega}$ rather than $\mathcal{L}$, and Section 2 exploits this fact to conclude that the e.c. dimension groups do not form an elementary class. Yet Section 3 shows that the schemas of Section 2 axiomatize the e.c. dimension groups. Because these schemas make every existential $\mathcal{L}$-formula equivalent in e.c. dimension groups to a quantifier-free formula of $\mathcal{L}_{\omega_{1} \omega}$, Section 4 can show that the class of dimension groups has the amalgamation property. The particular form of the schemas allows one to show that every nontrivial idea 5 in an e.c. dimension group is an e.c. dimension group, that every nontrivial dimension group is cofinal in an e.c. dimension group, and that the class of dimension groups with distinguished

Received by the editors January 2, 2010 and, in revised form, January 13, 2010 and May 23, 2010 .

2010 Mathematics Subject Classification. Primary 03C60, 06F20; Secondary 03C25.

${ }^{1}$ Any two elements have an upper bound.

${ }^{2}$ If $n$ is a positive integer for which $n x \geq 0$, then $x \geq 0$.

${ }^{3}$ If $x, y \leq z, v$, then there is $w$ with $x, y \leq w \leq z, v$.

${ }^{4}$ Every dimension group may be embedded in its divisible hull, and the usual proof of quantifier elimination for divisible totally ordered Abelian groups applies in divisible dimension groups to existential quantifications of conjunctions of atomic formulas.

${ }^{5}$ An ideal in a partially ordered Abelian group is a directed convex subgroup. 
order unit 6 has the amalgamation property. Exploiting results of [4, one may also show that the class of simple7 dimension groups with distinguished order unit has the amalgamation property. Section 5 determines the structure of quantifier-free $\mathcal{L}$-types that may be isolated by existential formulas, and Section 6 invokes this analysis to show both that there are $2^{\aleph_{0}}$ pairwise nonembeddable countable finitely generic dimension groups and that there is no algebraically prime e.c. dimension group. Though there are many isomorphism classes of finitely generic dimension groups, all are elementarily equivalent, and Section 6 reveals special properties that distinguish these groups, or infinitely generic dimension groups, among e.c. dimension groups. While some of these results suggest that e.c. dimension groups are difficult to build or to describe, Section 7 uses the operations exploited in Section 2 to build functors embedding nontrivial dimension groups into e.c. or even $\omega_{1}$-existentially saturated dimension groups. The Conclusion, finally, raises some questions not answered here.

Elliott [3] classifies AF $C^{*}$-algebras with the help of dimension groups. With any unital AF algebra $(B, 1)$, Elliott associates a (countable) dimension group $K_{0}(B)$ with order unit [1]; every countable dimension group with order unit arises in this way, and $(B, 1) \cong\left(B^{\prime}, 1^{\prime}\right)$ just in case $\left(K_{0}(B),[1]\right) \cong\left(K_{0}\left(B^{\prime}\right),\left[1^{\prime}\right]\right)$. Other modeltheoretic properties also correspond if on the AF-algebra side one uses the "logic of metric structures" of [1]. With respect to a certain metric language $\mathcal{M}$ for unital $C^{*}$-algebras and a new constant symbol $u$, the $\mathcal{M}$-structure $(B, 1)$ is algebraically (existentially) closed in the $\mathcal{M}$-structure $\left(B^{\prime}, 1^{\prime}\right)$ just in case the $\mathcal{L} \cup\{u\}$ structure $\left(K_{0}(B),[1]\right)$ is algebraically (existentially) closed in the $\mathcal{L} \cup\{u\}$-structure $\left(K_{0}\left(B^{\prime}\right),\left[1^{\prime}\right]\right)$ (see 9 for exact statement of these results). So algebraically (existentially) closed unital AF-algebras correspond to algebraically (existentially) closed dimension groups with distinguished order unit, and the possibility of building the former via the latter inspired the present work.

Model-theoretic notions and results exploited here will be be found mostly in [5]. In particular, if $\mathcal{A}$ is a structure for a first-order language $\mathcal{Q}, \mathcal{Q}_{A}$ is the expansion of $\mathcal{Q}$ by new constant symbols for elements of $\mathcal{A}$ 's domain $A$, and $\operatorname{diag}(\mathcal{A})$ is the diagram of $\mathcal{A}$ : the set of atomic and negated-atomic $\mathcal{Q}_{A}$-sentences true in $\mathcal{A}_{A}$, the natural expansion of $\mathcal{A}$ to a $\mathcal{Q}_{A}$-structure. More specialized model-theoretic results from [2] and [7] will be needed at the end of Section 6.

I am grateful to C. Wood for helpful discussions about e.c. structures.

\section{SOME PROPERTIES OF E.C. DIMENSION GROUPS}

A partially ordered Abelian group will be called nontrivial just in case it has at least one nonzero element. In a directed partially ordered Abelian group every element is the difference of two nonnegative elements: so if the group is nontrivial, it contains a strictly positive element. In the context of $\mathcal{L}$-formulas

$$
t<v
$$

will serve as an abbreviation for

$$
t \leq v \wedge t \neq v
$$

\footnotetext{
${ }^{6} \mathrm{An}$ order unit in a partially ordered Abelian group is a nonnegative element whose naturalnumber multiples are cofinal in the partial ordering.

${ }^{7} \mathrm{~A}$ dimension group is simple just in case it has no proper nonzero ideals.
} 
Because the study of e.c. dimension groups does not depend on whether the weak or strict partial ordering is represented by an atomic formula, dimension groups will often be regarded as structures for the language $\mathcal{L}^{<}=\{+,-,<, 0\}$, with

$$
t \leq v
$$

an abbreviation for

$$
t<v \vee t=v \text {. }
$$

Let $(G, \leq)$ be an e.c. dimension group. Since $(G, \leq)$ is algebraically closed, $G$ is divisible; and since $\{0\}$ embeds in $\mathbb{Q},(G, \leq)$ is nontrivial and contains a strictly positive element. Thus $(G, \leq)$ is strictly directed: that is, any $x, y \in G$ have a strict upper bound $z>x, y$.

Lemma 2.1. For each $i$ in the nonempty index set I, let the $\mathcal{L}^{<}$-structure $\mathcal{M}_{i}$ be a nontrivial dense ordered Abelian group: then the $\mathcal{L}^{<}$-produc $9^{9} \prod_{i \in I} \mathcal{M}_{i}$ is a dimension group that is strictly directed and satisfies the strict interpolation property

$$
x, y<z, v \rightarrow \exists w(x, y<w<z, v) .
$$

Proof. Each $\mathcal{M}_{i}$ has the desired properties, which may be expressed by Horn sentences of $\mathcal{L}^{<}$and so are preserved by the product.

This result is applied to dimension groups in combination with

Lemma 2.2. Let $(M, \leq)$ be a divisible partially ordered Abelian group with isolated order. If $a_{1}, \ldots, a_{n} \in M$ and

$$
0 \not \leq \sum_{i} m_{i} a_{i}
$$

whenever $\bar{m} \in \mathbb{N}^{n} \backslash\{\overline{0}\}$, then there is $\preccurlyeq \subseteq M^{2}$, with $\preccurlyeq \supseteq \leq$, such that $(M, \preccurlyeq)$ is an ordered Abelian group in which $a_{1}, \ldots, a_{n} \prec 0$.

Proof. Because $(M, \leq)$ is divisible with isolated order, elements $x$ of $M$ have uniquely determined rational multiples $q x$ for all $q \in \mathbb{Q}$. If $\bar{q} \in \mathbb{Q}^{n}$ is nonnegativei.e., all its entries are nonnegative - and

$$
0 \leq \sum_{i} q_{i} a_{i}
$$

then there are $\bar{m} \in \mathbb{N}^{n}$ and $N>0$ in $\mathbb{N}$ with

$$
\bar{q}=\bar{m} / N,
$$

and

$$
0 \leq N \sum_{i} q_{i} a_{i}=\sum_{i} m_{i} a_{i}
$$

$\bar{m}=\overline{0}$, and $\bar{q}=\overline{0}$. Thus

$$
0 \not \leq \sum_{i} q_{i} a_{i}
$$

whenever $\overline{0} \leq \bar{q} \in \mathbb{Q}^{n} \backslash\{\overline{0}\}$.

Let

$$
S=\left\{p-\sum_{i} q_{i} a_{i}: 0 \leq p \text { and } \overline{0} \leq \bar{q} \in \mathbb{Q}^{n}\right\} .
$$

\footnotetext{
${ }^{8}$ In what follows, "ordered Abelian group" means "totally ordered Abelian group".

${ }^{9}$ The product partially ordered in this way appears in (4 , p. 6).
} 
$0 \in S$, which is closed under addition and nonnegative rational multiplication and contains all nonnegative elements of $M$. If $r \in S \cap(-S)$, then for $i=1,2$ there are $p_{i} \geq 0$ in $M$ and $q_{i j} \geq 0$ in $\mathbb{Q}(j=1, \ldots, n)$ with

$$
r=p_{1}-\sum_{j} q_{1 j} a_{j}=-\left(p_{2}-\sum_{j} q_{2 j} a_{j}\right) .
$$

So

$$
0 \leq p_{1}+p_{2}=\sum_{j}\left(q_{1 j}+q_{2 j}\right) a_{j},
$$

and since all the $q$ 's are nonnegative rationals, $q_{1 j}+q_{2 j}=0$ by the remarks in the first paragraph and $q_{1 j}=0=q_{2 j}$. Thus $r=p_{1}=-p_{2}$ is both nonnegative and nonpositive and $S \cap(-S)=\{0\}$.

Invoke Zorn's Lemma to obtain $P \subseteq M$ maximal with respect to inclusion among all $T \subseteq M$ such that $S \subseteq T, T+T \subseteq T, q T \subseteq T$ when $0 \leq q \in \mathbb{Q}$, and $T \cap(-T)=$ $\{0\}$. If $P \cup(-P)=M$, then $P$ will be the nonnegative cone of an ordering $\preccurlyeq$, with $\leq \subseteq \preccurlyeq$, making $(M, \preccurlyeq)$ an ordered Abelian group, and since $-a_{1}, \ldots,-a_{n} \in P$ and each $a_{i} \neq 0$ by hypothesis, $a_{1}, \ldots, a_{n} \prec 0$ as desired. If $y \in M \backslash P$, then $P \subset P+\{q y: 0 \leq q \in \mathbb{Q}\}$, and the maximality of $P$ implies that there are $p_{1}, p_{2} \in P$ and $q_{1}, q_{2} \geq 0$ in $\mathbb{Q}$ with

$$
0 \neq p_{1}+q_{1} y=-\left(p_{2}+q_{2} y\right) .
$$

Thus

$$
\left(q_{1}+q_{2}\right) y=-\left(p_{1}+p_{2}\right) .
$$

$q_{1}+q_{2} \geq 0$. If $q_{1}+q_{2}=0$, then $q_{1}=q_{2}=0$ and

$$
0 \neq p_{1}=-p_{2},
$$

contrary to $P \cap(-P)=\{0\}$. Thus $q_{1}+q_{2}>0$ and

$$
y=-\frac{1}{q_{1}+q_{2}}\left(p_{1}+p_{2}\right) \in-P .
$$

So $M \backslash P \subseteq-P$ and the argument is complete.

One may now state

Theorem 2.3. The e.c. dimension group $(G, \leq)$ obeys

(i) Strict interpolation.

(ii) If $a_{1}, \ldots, a_{n} \in G$ and

$$
0 \not \leq \sum_{i} m_{i} a_{i}
$$

whenever $\bar{m} \in \mathbb{N}^{n} \backslash\{\overline{0}\}$, then there is $y \in G$ with

$$
\bigwedge_{i} a_{i}<y \wedge 0 \nless y \text {. }
$$

Proof. Let

$$
\mathcal{O}(G)=\left\{\prec \subseteq G^{2}: \leq \subseteq \preccurlyeq \text { and }(G, \prec) \text { is an ordered Abelian group }\right\} .
$$

Because $G$ is a nontrivial divisible group, the $\mathcal{L}^{<}$-structure $(G, \prec)$ is a nontrivial dense ordered Abelian group whenever $\prec \in \mathcal{O}(G)$. Since $G$ contains negative elements, Lemma 2.2 makes $\mathcal{O}(G)$ nonempty; so by Lemma 2.1, $\widehat{G}=\prod_{\prec \in \mathcal{O}(G)}(G, \prec)$ is a nontrivial dimension group that satisfies strict interpolation. One easily checks that the diagonal map $\delta: G \rightarrow \widehat{G}$ sending each $g \in G$ to the constant function with 
value $g$ is an $\mathcal{L}^{<}$-embedding; so since $(G, \leq)$ is existentially closed, it satisfies strict interpolation.

The hypothesis of (ii), and Lemma 2.2 provide orderings $\prec$ in $\mathcal{O}(G)$ with $a_{1}, \ldots, a_{n} \prec 0$; whenever $\prec$ has this property,

$$
a_{1}, \ldots, a_{n} \prec \frac{1}{2} \max _{\prec}\left\{a_{1}, \ldots, a_{n}\right\} \prec 0
$$

in the divisible ordered Abelian group $(G, \prec)$. Since $(G, \leq)$ is strictly directed, there is $c \in G$ with $a_{1}, \ldots, a_{n}<c$. If $y$ is the element of $\widehat{G}$ that sends each $\prec \in \mathcal{O}(G)$ with $a_{1}, \ldots, a_{n} \prec 0$ to $\frac{1}{2} \max _{\prec}\left\{a_{1}, \ldots, a_{n}\right\}$ and every other $\prec \in \mathcal{O}(G)$ to $c$, then

$$
\bigwedge_{i} \delta\left(a_{i}\right)<y \wedge 0 \nless y
$$

in $\widehat{G}$. Because $G$ is existentially closed, the desired conclusion follows.

A simple argument shows that the converse of (ii) holds in any partially ordered Abelian group with isolated order.

Before describing other properties of e.c. dimension groups, one may exploit 2.3(ii) to show that

Corollary 2.4. The e.c. dimension groups do not form an elementary class.

Proof. Suppose otherwise, and let $T$ be a set of $\mathcal{L}^{<}$-axioms for this elementary class. By 2.3(ii) 10

$$
T \models \forall x_{1}, x_{2}\left(\bigwedge_{\overline{0} \neq \bar{m} \in \mathbb{N}^{2}} 0 \not \leq m_{1} x_{1}+m_{2} x_{2} \rightarrow \exists y\left(x_{1}, x_{2}<y \wedge 0 \nless y\right)\right) .
$$

So the compactness theorem provides a finite $U \subseteq \mathbb{N}^{2} \backslash\{\overline{0}\}$ with

$$
T \models \forall x_{1}, x_{2}\left(\bigwedge_{\bar{m} \in U} 0 \not \leq m_{1} x_{1}+m_{2} x_{2} \rightarrow \exists y\left(x_{1}, x_{2}<y \wedge 0 \nless y\right)\right) .
$$

Let $k>0$ be a strict upper bound on the entries of ordered pairs in $U$.

The additive Abelian group $\mathbb{Q}(\sqrt{2})$ may be partially ordered so that for $a, b \in \mathbb{Q}$,

$$
0 \sqsubset a+b \sqrt{2} \text { iff } 0<a \pm b \sqrt{2} \text { in } \mathbb{R} .
$$

One easily sees that $\sqsubset$ makes $\mathbb{Q}(\sqrt{2})$ a partially ordered group with directed, isolated order. Assume, for the moment,

Lemma 2.5. $(\mathbb{Q}(\sqrt{2}), \sqsubset)$ satisfies strict interpolation, and so is a dimension group.

Consider the elements $\sqrt{2}$ and $-k^{-1} \sqrt{2}$ of $\mathbb{Q}(\sqrt{2})$. If $(m, n) \in U$ and

$$
0 \sqsubseteq m \sqrt{2}+n\left(-k^{-1} \sqrt{2}\right),
$$

then $0 \sqsubseteq\left(m-n k^{-1}\right) \sqrt{2}, 0 \leq \pm\left(m-n k^{-1}\right) \sqrt{2}$ in $\mathbb{R}$, and

$$
m=n k^{-1} ; \quad \therefore k m=n \text {. }
$$

If $m>0, n=k m \geq k>n$; so $m=0=n$, though $(0,0) \notin U$. This contradiction implies that

$$
\bigwedge_{(m, n) \in U} 0 \nsubseteq m \sqrt{2}+n\left(-k^{-1} \sqrt{2}\right)
$$

\footnotetext{
${ }^{10}$ In what follows, infinite conjunctions or disjunctions are indicated by explicit reference to an infinite index set; so a conjunction or disjunction without such explicit reference is finite.
} 
in $(\mathbb{Q}(\sqrt{2}), \sqsubset)$ and in any partially ordered group containing $(\mathbb{Q}(\sqrt{2}), \sqsubset)$. Since $(\mathbb{Q}(\sqrt{2}), \sqsubset)$ is a dimension group (Lemma 2.5) it may be embedded in an e.c. dimension group $(H, \triangleleft)$, and in this model of $T$ there is an element $a$ with

$$
\sqrt{2},-k^{-1} \sqrt{2} \triangleleft a \wedge 0 丸 a .
$$

On the other hand,

$$
1 \sqrt{2}+k\left(-k^{-1} \sqrt{2}\right)=0
$$

in $(\mathbb{Q}(\sqrt{2}), \sqsubset) \subseteq(H, \triangleleft)$; so if $\sqrt{2},-k^{-1} \sqrt{2} \triangleleft a$, then

$$
0=1 \sqrt{2}+k\left(-k^{-1} \sqrt{2}\right) \triangleleft 1 a+k a=(1+k) a
$$

and $0 \triangleleft a$ because $(H, \triangleleft)$ has isolated order. This contradiction implies that there is no set $T$ of $\mathcal{L}^{<}$-axioms for the class of e.c. dimension groups.

To prove Lemma 2.5. consider the partially ordered group $(F, 4)$ of all linear functions

$$
x \in[-1,1] \mapsto a+b \sqrt{2} x
$$

with $a, b \in \mathbb{Q}$, where for $g, h \in F$,

$$
g \triangleleft h \text { iff } g(x)<h(x) \text { for all } x \in[-1,1] .
$$

Since elements of $\mathbb{Q}(\sqrt{2})$ are uniquely of the form $a+b \sqrt{2}$ with $a, b \in \mathbb{Q}$, one may define $\varphi: \mathbb{Q}(\sqrt{2}) \rightarrow F$ by

$$
\varphi(a+b \sqrt{2})=(x \in[-1,1] \mapsto a+b \sqrt{2} x),
$$

and one easily checks that $\varphi:(\mathbb{Q}(\sqrt{2}), \sqsubset) \cong(F, \varangle)$. So the desired result holds if $(F, \varangle)$ obeys strict interpolation.

If $f, g \triangleleft h, k$ in $(F, \varangle)$, then

$$
f(-1), g(-1)<h(-1), k(-1) \text { and } f(1), g(1)<h(1), k(1)
$$

in the divisible ordered group $\mathbb{Q}(\sqrt{2}) \subseteq \mathbb{R}$. If $\alpha, \beta \in \mathbb{Q}(\sqrt{2})$ obey

$$
f(-1), g(-1)<\alpha<h(-1), k(-1) \text { and } f(1), g(1)<\beta<h(1), k(1)
$$

and $l$ is the unique linear function on $[-1,1]$ with $l(-1)=\alpha$ and $l(1)=\beta$, then

$$
f(x), g(x)<l(x)<h(x), k(x) \text { for all } x \in[-1,1] .
$$

Since $\mathbb{Q}$ is dense in $\mathbb{Q}(\sqrt{2})$ there is a sequence $\left\{q_{n}\right\}_{n \geq 1}$ from $\mathbb{Q}$ that converges to $l(0)$; if for $n \geq 1, l_{n}$ is the unique linear function on $[-1,1]$ with $l_{n}(0)=q_{n}$ and $l_{n}(1)=l(1)$, one sees that $\left\{l_{n}\right\}_{n \geq 1}$ converges uniformly on $[-1,1]$ to $l$. So without loss of generality, $l(0) \in \mathbb{Q}$. Since $\mathbb{Q} \cdot \sqrt{2}$ is dense in $\mathbb{Q}(\sqrt{2})$ there is a sequence $\left\{r_{n}\right\}_{n \geq 1}$ from $\mathbb{Q} \cdot \sqrt{2}$ that converges to $l(1)-l(0)$; if

$$
m_{n}(x)=l(0)+r_{n} x \text { for all } x \in[-1,1],
$$

then each $m_{n} \in F$ and $\left\{m_{n}\right\}_{n \geq 1}$ converges uniformly on $[-1,1]$ to $l$. Thus $f, g$ $m_{n} \triangleleft h, k$ for some $n$ and the argument is complete.

In $\mathcal{L}$ or $\mathcal{L}^{<}$

will abbreviate

$$
t \mid v
$$

$$
t \nless v \wedge t \neq v \wedge v \nless t:
$$

$t$ is incomparable with $v$. 
Viewing the e.c. dimension group $G$ as an $\mathcal{L}^{<}$-structure, consider the $\mathcal{L}^{<}$-power

$$
\left(G^{\aleph_{0}},<^{\aleph_{0}}\right)=\prod_{i \in \mathbb{N}}(G,<)
$$

of $(G,<)$. Because one may express with Horn sentences the properties of being divisible, being strictly directed, and obeying strict interpolation, these properties transfer from $(G,<)$ to $\left(G^{\aleph_{0}},<^{\aleph_{0}}\right)$. Much as before, the diagonal map $\delta: G \rightarrow G^{\aleph_{0}}$ embeds $(G,<)$ into $\left(G^{\aleph_{0}},<\aleph^{\aleph_{0}}\right)$.

Theorem 2.6. The e.c. dimension group $(G,<)$ obeys the following first-order schemas of $\mathcal{L}^{<}$(where the variable $y$ does not occur in the terms $a_{i}, b_{j}$, and $w_{s}$ and $\varphi$ is a conjunction of inequalities in the distinct variables $\bar{y}=\left(y_{s}\right)_{s \in S}$ and $\left.\bar{x}=\left(x_{t}\right)_{t \in T}\right):$

(i) $\bigwedge_{s} \exists y\left(\bigwedge_{i} a_{i}<y \wedge \bigwedge_{j} y<b_{j} \wedge y \mid w_{s}\right) \rightarrow \exists y\left(\bigwedge_{i} a_{i}<y \wedge \bigwedge_{j} y<b_{j} \wedge \bigwedge_{s} y \mid w_{s}\right)$.

(ii) $\bigwedge_{s} \exists \bar{y}\left(\varphi \wedge y_{s} \mid 0\right) \rightarrow \exists \bar{y}\left(\varphi \wedge \bigwedge_{s} y_{s} \mid 0\right)$.

(iii) $\bigwedge_{i} a_{i}<0 \wedge \bigwedge_{j} 0<b_{j} \rightarrow \exists y\left(\bigwedge_{i} a_{i}<y \wedge \bigwedge_{j} y<b_{j} \wedge y \mid 0\right)$.

Proof. (i)-(iii) are all established in the same way.

Suppose that for each $s \in S$,

$$
\bigwedge_{i} a_{i}<y_{s} \wedge \bigwedge_{j} y_{s}<b_{j} \wedge y_{s} \mid w_{s} \text { in }(G,<) .
$$

Let the $m$ elements of $S$ be $s_{0}, \ldots, s_{m-1}$. If $y \in G^{\aleph_{0}}$ is given by

$$
y_{l}=y_{s_{r}} \text { if } r<m \text { and } l \equiv r \bmod m,
$$

then in $\left(G^{\aleph_{0}},<\aleph^{\aleph_{0}}\right)$,

$$
\bigwedge_{i} \delta\left(a_{i}\right)<y \wedge \bigwedge_{j} y<\delta\left(b_{j}\right) \wedge \bigwedge_{s} y \mid \delta\left(w_{s}\right) .
$$

Because $(G,<)$ is existentially closed it obeys the consequent of (i).

Let $\varphi=\varphi\left(x_{t_{0}}, \ldots, x_{t_{n-1}}, y_{s_{0}}, \ldots, y_{s_{m-1}}\right)$, and suppose that for each $i<m$ there are $b_{0}^{(i)}, \ldots, b_{m-1}^{(i)} \in G$ such that

$$
\varphi\left(a_{0}, \ldots, a_{n-1}, b_{0}^{(i)}, \ldots, b_{m-1}^{(i)}\right) \wedge b_{i}^{(i)} \mid 0
$$

in $(G,<)$. For $j<m$ let $c_{j} \in G^{\aleph_{0}}$ be given by

$$
c_{j l}=b_{j}^{(r)} \text { if } r<m \text { and } l \equiv r \bmod m ;
$$

then in $\left(G^{\aleph_{0}},<^{\aleph_{0}}\right)$,

$$
\varphi\left(\delta\left(a_{0}\right), \ldots, \delta\left(a_{n-1}\right), c_{0}, \ldots, c_{m-1}\right) \wedge \bigwedge_{s} c_{s} \mid 0
$$

Because $(G,<)$ is existentially closed it obeys the consequent of (ii).

Finally, suppose that

$$
\bigwedge_{i} a_{i}<0 \wedge \bigwedge_{j} 0<b_{j}
$$

in $(G,<)$. Strict interpolation, or the property of being strictly directed, provides $c, d \in G$ with

$$
\bigwedge_{i} a_{i}<c<0 \wedge \bigwedge_{j} 0<d<b_{j}
$$


If $y \in G^{\aleph_{0}}$ is given by

$$
y_{2 l}=c \text { and } y_{2 l+1}=d,
$$

then

$$
\bigwedge_{i} \delta\left(a_{i}\right)<y \wedge \bigwedge_{j} y<\delta\left(b_{j}\right) \wedge y \mid 0
$$

in $\left(G^{\aleph_{0}},<\aleph^{\aleph_{0}}\right)$. Because $(G,<)$ is existentially closed it obeys the consequent of (iii).

The class of nontrivial dimension groups with distinguished order unit, though not elementary, is inductive, and so every such dimension group may be embedded in another, with the same distinguished order unit, that is existentially closed with respect to this class. Structures in this class are naturally viewed as structures for the language $\mathcal{L}^{<} \cup\{u\}$, where $u$ is a new constant symbol naming the distinguished order unit. Arguments like those given above show that every nontrivial dimension group, with distinguished order unit, existentially closed among dimension groups of this kind is divisible and obeys the schemas examined in Theorems 2.3 and 2.6 . The proofs change only in replacing $\widehat{G}\left(G^{\aleph_{0}}\right)$ by the convex hull in $\widehat{G}\left(G^{\aleph_{0}}\right)$ of $\delta(G)$, where $\delta: G \rightarrow \widehat{G}\left(\delta: G \rightarrow G^{\aleph_{0}}\right)$ is the diagonal map.

\section{A Characterization of E.C. Dimension Groups}

One may show that e.c. dimension groups are characterized by the schemas discovered in Section 2 by using them to convert existential $\mathcal{L}^{<}$-formulas to quantifier-

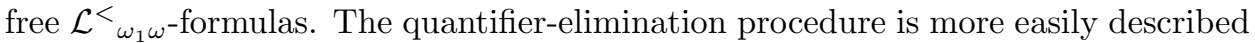
if one uses a language, extending $\mathcal{L}^{<}$, that takes advantage of the fact that e.c. dimension groups are divisible. Let

$$
\mathcal{Q}=\mathcal{L}^{<} \cup\{r \cdot: r \in \mathbb{Q}\},
$$

where each $r$. is a new unary function symbol. If $T^{<}$is the $\mathcal{L}^{<}$-theory of divisible dimension groups and $T^{\mathcal{Q}}$ is the $\mathcal{Q}$-theory of divisible dimension groups - with $r$. representing scalar multiplication by $r \in \mathbb{Q}$ - then $T^{\mathcal{Q}} \models T^{<}$, every model of $T^{<}$ expands uniquely to a model of $T^{\mathcal{Q}}$, every atomic $\mathcal{Q}$-formula is $T^{\mathcal{Q}}$-equivalent to an atomic $\mathcal{L}^{<}$-formula, and the e.c. dimension groups are the e.c. models of $T^{<}$, which when viewed as $\mathcal{Q}$-structures are the e.c. models of $T^{\mathcal{Q}}$. In what follows $\mathcal{Q}$ will supplant $\mathcal{L}^{<}$wherever convenient.

Section 2's schemas - in which initial universal quantifiers are suppressed-run as follows:

(1) $\exists z(x, y<z)$

(2) $x, y<z, v \rightarrow \exists w(x, y<w<z, v)$

(3) $\bigwedge_{\bar{m} \in \mathbb{N}^{n} \backslash\{\overline{0}\}} 0 \not \leq \sum_{i} m_{i} a_{i} \rightarrow \exists y\left(\bigwedge_{i} a_{i}<y \wedge 0 \nless y\right)$

(4) $\bigwedge_{s} \exists y\left(\bigwedge_{i} a_{i}<y \wedge \bigwedge_{j} y<b_{j} \wedge y \mid w_{s}\right) \rightarrow \exists y\left(\bigwedge_{i} a_{i}<y \wedge \bigwedge_{j} y<b_{j} \wedge \bigwedge_{s} y \mid w_{s}\right)$

(5) $\bigwedge_{s} \exists \bar{y}\left(\varphi \wedge y_{s} \mid 0\right) \rightarrow \exists \bar{y}\left(\varphi \wedge \wedge_{s} y_{s} \mid 0\right)$

(6) $\bigwedge_{i} a_{i}<0 \wedge \bigwedge_{j} 0<b_{j} \rightarrow \exists y\left(\bigwedge_{i} a_{i}<y \wedge \bigwedge_{j} y<b_{j} \wedge y \mid 0\right)$

So formulas from schema (3) are infinitary formulas of $\mathcal{Q}_{\omega_{1} \omega}$, while formulas from the other schemas are first-order $\mathcal{Q}$-formulas. The converses of (2)-(5) are true in any partially ordered Abelian group with isolated order. When $I \subseteq\{1, \ldots, 6\}$ let $T_{I}$ be the expansion of $T^{\mathcal{Q}}$ by the schemas $(j)$ with $j \in I$. Thus $T_{I}$ is a first-order theory exactly when $3 \notin I$. 
Lemma 3.1. Every quantifier-free formuld 11 is $T^{\mathcal{Q}}$-equivalent to a positive propositional combination of identities $t=v$, inequalities $t<v$, and incomparabilities $t \mid v$.

Proof. The argument relies merely on the axioms of $T^{\mathcal{Q}}$ guaranteeing a partial ordering. Every quantifier-free formula is logically equivalent to a positive propositional combination of atomic and negated-atomic $\mathcal{Q}$-formulas: so the desired result holds if negations of atomic $\mathcal{Q}$-formulas are $T^{\mathcal{Q}}$-equivalent to formulas of the given kind. The formula

$$
t=v \vee t<v \vee v<t \vee t \mid v
$$

is logically valid, and $T^{\mathcal{Q}}$ implies that the disjuncts are mutually exclusive: so negations of atomic formulas have the desired property. For example, $t \neq v$ is $T^{\mathcal{Q}}$-equivalent to $t<v \vee v<t \vee t \mid v$.

A simple argument exploiting the vector-space axioms in $T^{\mathcal{Q}}$ shows that

Lemma 3.2. $T^{\mathcal{Q}}$ implies that

$$
t \square v \leftrightarrow t+w \square v+w
$$

and that

$$
t \square v \leftrightarrow q t \square q v
$$

when $\square \in\{=,<, \mid\}$ and $0<q \in \mathbb{Q}$.

Since $\exists$ commutes with $\vee$, Lemma 3.1 reduces the study of existential $\mathcal{Q}$-formulas to the study of existential quantifications of conjunctions of identities, inequalities, and incomparabilities. The following result brings the goal much closer:

Lemma 3.3. Let $\varphi(\bar{x}, \bar{y})$ be a finite conjunction of identities, inequalities, and incomparabilities in $\bar{x}=\left(x_{1}, \ldots, x_{k}\right)$ and $\bar{y}=\left(y_{1}, \ldots, y_{l}\right) . \exists \bar{y} \varphi$ is $T_{1,2,4,5}$-equivalent to a finite conjunction $\gamma(\bar{x})$ of identities, inequalities, incomparabilities, and formulas

$$
\exists z(\theta \wedge z \mid 0)
$$

in which $\theta$ is a finite conjunction of inequalities in $\bar{x}$ and the new variable $z$. One may assume that variables $x_{i}$ occurring only in inequalities of $\varphi$ occur only in inequalities of $\gamma$-perhaps inside some $\theta$-and that $\gamma$ contains identities only if $\varphi$ does.

Proof. The proof goes by induction on $l \geq 1$. Lemma 3.2 allows one to assume that $\varphi$ is

$$
\bigwedge_{i} a_{i}<y_{l} \wedge \bigwedge_{j} y_{l}<b_{j} \wedge \bigwedge_{m} y_{l}=c_{m} \wedge \bigwedge_{s} y_{l} \mid w_{s} \wedge \psi
$$

where the $a \mathrm{~s}, b \mathrm{~s}, c \mathrm{~s}$, and $w \mathrm{~s}$ are $\mathcal{Q}$-terms in $\bar{x}, y_{1}, \ldots, y_{l-1}$ and $\psi$ is a conjunction of identities, inequalities, and incomparabilities in $\bar{x}, y_{1}, \ldots, y_{l-1}$. If $\varphi=\psi, \exists \bar{y} \varphi$ is equivalent to $\exists y_{1} \ldots \exists y_{l-1} \varphi$ and the induction hypothesis gives the desired result: so assume that $\varphi \neq \psi$.

If $m_{0} \in M$, the index set for the third conjunct of $\varphi$, then $\exists \bar{y} \varphi$ is equivalent to

$$
\exists y_{1} \ldots \exists y_{l-1}\left(\bigwedge_{i} a_{i}<c_{m_{0}} \wedge \bigwedge_{j} c_{m_{0}}<b_{j} \wedge \bigwedge_{m} c_{m_{0}}=c_{m} \wedge \bigwedge_{s} c_{m_{0}} \mid w_{s} \wedge \psi\right),
$$

and any $x_{i}$ occurring only in inequalities of $\varphi$ occurs only in inequalities of the quantifier-free part of this formula. So the induction hypothesis completes the

\footnotetext{
${ }^{11}$ Formulas are first-order formulas unless specified otherwise.
} 
argument if $M \neq \emptyset$, and one may assume that $M=\emptyset$. Thus the only identities in $\varphi$ are in $\psi$.

Schema (4) makes $\exists y_{l} \varphi$ equivalent to

$$
\bigwedge_{s} \exists y_{l}\left(\bigwedge_{i} a_{i}<y_{l} \wedge \bigwedge_{j} y_{l}<b_{j} \wedge y_{l} \mid w_{s}\right) \wedge \psi
$$

and any $x_{i}$ occurring only in inequalities of $\varphi$ occurs only in the terms $a, b$ or in inequalities that are conjuncts of $\psi$. Lemma 3.2 makes the displayed formula equivalent to

$$
\bigwedge_{s} \exists y_{l}\left(\bigwedge_{i} a_{i}-w_{s}<y_{l} \wedge \bigwedge_{j} y_{l}<b_{j}-w_{s} \wedge y_{l} \mid 0\right) \wedge \psi,
$$

and any $x_{i}$ occurring only in inequalities of $\varphi$ occurs only in the terms $a_{i}-w_{s}$, $b_{j}-w_{s}$, or in inequalities that are conjuncts of $\psi$. If $l=1$ the argument is complete: so assume $l>1$.

For each $s \in S$ let $y_{l s}$ be a new variable. The last displayed formula is logically equivalent to

$$
\bigwedge_{s} \exists y_{l s}\left(\bigwedge_{i} a_{i}-w_{s}<y_{l s} \wedge \bigwedge_{j} y_{l s}<b_{j}-w_{s} \wedge y_{l s} \mid 0\right) \wedge \psi
$$

and to

$$
\left(\exists y_{l s}\right)_{s \in S}\left(\bigwedge_{s} \bigwedge_{i} a_{i}-w_{s}<y_{l s} \wedge \bigwedge_{j} y_{l s}<b_{j}-w_{s} \wedge y_{l s} \mid 0 \wedge \psi\right) .
$$

By the induction hypothesis,

$$
\exists y_{1} \ldots \exists y_{l-1}\left(\bigwedge_{s} \bigwedge_{i} a_{i}-w_{s}<y_{l s} \wedge \bigwedge_{j} y_{l s}<b_{j}-w_{s} \wedge \psi\right)
$$

is $T_{1,2,4,5}$-equivalent to a conjunction $\gamma$ of identities, inequalities, incomparabilities, and formulas $\exists z(\theta \wedge z \mid 0)$ with $\theta$ a conjunction of inequalities; $\gamma$ contains identities only if $\psi$ does; and each $y_{l s}$, as well as each $x_{i}$ occurring only in inequalities of $\varphi$, occurs only in inequalities of $\gamma$ (including perhaps conjuncts of various $\theta \mathrm{s}$ ). So $\exists \bar{y} \varphi$ is $T_{1,2,4,5}$-equivalent to

$$
\left(\exists y_{l s}\right)_{s \in S}\left(\gamma \wedge \bigwedge_{s} y_{l s} \mid 0\right) .
$$

Using distinct variables $z$ for the conjuncts $\exists z(\theta \wedge z \mid 0)$ of $\gamma$, one may assume that the last displayed formula is

$$
\left(\exists y_{l s}\right)_{s \in S}\left(\alpha \wedge \bigwedge_{m} \exists z_{m}\left(\theta_{m} \wedge z_{m} \mid 0\right) \wedge \beta \wedge \bigwedge_{s} y_{l s} \mid 0\right),
$$

where $\alpha$ and the $\theta$ s are conjunctions of inequalities, $z_{m}$ occurs neither in $\alpha$ nor in any $\theta_{n}$ with $n \neq m, \beta$ is a conjunction of identities and incomparabilities in which no $y_{l s}$ or $z_{m}$ occurs, $\beta$ contains identities only if $\psi$ does, and each $x_{i}$ occurring only in inequalities of $\varphi$ occurs only in $\alpha$ or in some $\theta$. So the last displayed formula is logically equivalent to

$$
\left(\exists y_{l s}\right)_{s \in S} \exists \bar{z}\left(\alpha \wedge \bigwedge_{m} \theta_{m} \wedge \bigwedge_{s} y_{l s}\left|0 \wedge \bigwedge_{m} z_{m}\right| 0\right) \wedge \beta .
$$

We simplify the notation by writing the first conjunct as

$$
\left(\exists v_{t}\right)_{t \in T}\left(\theta \wedge \bigwedge_{t} v_{t} \mid 0\right)
$$


where the $v$ s consist of the $y_{l s} \mathrm{~s}$ and $z \mathrm{~s}$ and $\theta$ is $\alpha \wedge \bigwedge_{m} \theta_{m}$. Schema (5) makes the last displayed formula equivalent to

$$
\bigwedge_{t} \exists \bar{v}\left(\theta \wedge v_{t} \mid 0\right)
$$

and so to

$$
\bigwedge_{t}\left(\exists v_{t}\right)\left(\left(\exists v_{r}\right)_{r \neq t} \theta \wedge v_{t} \mid 0\right) .
$$

$T_{1,2}$ makes each $\left(\exists v_{r}\right)_{r \neq t} \theta$ equivalent to a conjunction of inequalities; so the argument is complete.

Lemma 3.3 thus reduces the study of existential $\mathcal{Q}$-formulas to the study of formulas $\exists z(\theta \wedge z \mid 0)$, where $\theta$ is a conjunction of inequalities. If $z$ does not occur in $\theta$, then $\exists z(\theta \wedge z \mid 0)$ is logically equivalent to $\theta \wedge \exists z(z \mid 0)$, which by schemas (1) and (6) is equivalent to $\theta$. If $z$ does occur in $\theta$, then by Lemma 3.2 one may assume that $\theta$ is

$$
\bigwedge_{i} a_{i}<z \wedge \bigwedge_{j} z<b_{j}
$$

where the $a$ s and $b$ s are $\mathcal{Q}$-terms not containing $z$.

Lemma 3.4. $\forall z\left(\bigwedge_{i} a_{i}<z \wedge \bigwedge_{j} z<b_{j} \rightarrow z \nmid 0\right)$ is $T_{1,2,6}$-equivalent to

$$
\left(\bigwedge_{i, j} a_{i}<b_{j}\right) \rightarrow\left[\forall z\left(\bigwedge_{i} a_{i}<z \rightarrow 0<z\right) \vee \forall z\left(\bigwedge_{j} z<b_{j} \rightarrow z<0\right)\right] .
$$

Proof. Suppose the displayed formula holds. If

$$
\bigwedge_{i} a_{i}<z \wedge \bigwedge_{j} z<b_{j}
$$

then $\bigwedge_{i, j} a_{i}<b_{j}$, and by hypothesis,

$$
\forall z\left(\bigwedge_{i} a_{i}<z \rightarrow 0<z\right) \vee \forall z\left(\bigwedge_{j} z<b_{j} \rightarrow z<0\right) ;
$$

if, for example, the first disjunct holds, then $0<z$ and $z \nmid 0$.

Now assume that

$$
\forall z\left(\bigwedge_{i} a_{i}<z \wedge \bigwedge_{j} z<b_{j} \rightarrow z \nmid 0\right) \wedge \bigwedge_{i, j} a_{i}<b_{j} .
$$

By schema (6),

$$
\neg\left(\bigwedge_{i} a_{i}<0 \wedge \bigwedge_{j} 0<b_{j}\right) .
$$

So

$$
\forall z\left(\bigwedge_{i} a_{i}<z \wedge \bigwedge_{j} z<b_{j} \rightarrow z<0 \vee 0<z\right) .
$$

If there are $z, w$ with

$$
\bigwedge_{i} a_{i}<z, w \wedge \bigwedge_{j} z, w<b_{j} \wedge z<0 \wedge 0<w,
$$

then $\bigwedge_{i} a_{i}<0 \wedge \bigwedge_{j} 0<b_{j}$; so

$$
\forall z, w\left(\bigwedge_{i} a_{i}<z, w \wedge \bigwedge_{j} z, w<b_{j} \rightarrow z \nless 0 \vee 0 \nless w\right) .
$$


Because $\bigwedge_{i, j} a_{i}<b_{j}$, schemas (1) and (2) imply that

$$
\forall z\left(\bigwedge_{i} a_{i}<z \wedge \bigwedge_{j} z<b_{j} \rightarrow z<0\right) \vee \forall z\left(\bigwedge_{i} a_{i}<z \wedge \bigwedge_{j} z<b_{j} \rightarrow 0<z\right) .
$$

If the first disjunct holds and

$$
\bigwedge_{j} z<b_{j}
$$

schemas (1) and (2) provide $w$ with

$$
\bigwedge_{i} a_{i}<w \wedge z<w \wedge \bigwedge w<b_{j}
$$

and so $w<0$ by hypothesis and $z<0$. Thus

$$
\forall z\left(\bigwedge_{j} z<b_{j} \rightarrow z<0\right)
$$

follows from the first disjunct, and

$$
\forall z\left(\bigwedge_{i} a_{i}<z \rightarrow 0<z\right)
$$

follows from the second.

Thus $\exists z\left(\bigwedge_{i} a_{i}<z \wedge \bigwedge_{j} z<b_{j} \wedge z \mid 0\right)$ is $T_{1,2,6}$-equivalent to

$$
\bigwedge_{i, j} a_{i}<b_{j} \wedge \exists z\left(\bigwedge_{i} a_{i}<z \wedge 0 \nless z\right) \wedge \exists z\left(\bigwedge_{j} z<b_{j} \wedge z \nless 0\right)
$$

and to

$$
\bigwedge_{i, j} a_{i}<b_{j} \wedge \exists z\left(\bigwedge_{i} a_{i}<z \wedge 0 \nless z\right) \wedge \exists w\left(\bigwedge_{j}-b_{j}<w \wedge 0 \nless w\right) .
$$

So with the help of schema (3) one concludes that

Theorem 3.5. Any existential $\mathcal{Q}$-formula is $T_{1, \ldots, 6}$-equivalent to a quantifier-free formula of $\mathcal{Q}_{\omega_{1} \omega}$.

Thus

Corollary 3.6. The e.c. models of $T^{\mathcal{Q}}$ are the models of $T_{1, \ldots, 6}$.

Proof. The results of Section 2 show that every e.c. model of $T^{\mathcal{Q}}$ is a model of $T_{1, \ldots, 6}$. Conversely, suppose $\mathcal{M} \models T_{1, \ldots, 6}$, and let $\mathcal{N} \supseteq \mathcal{M}$ be a dimension group in which an existential $\mathcal{Q}_{M}$-sentence $\psi$ is true. $\psi=\exists \bar{y} \varphi(\bar{m}, \bar{y})$, where the $m$ s come from $M$ and $\varphi(\bar{x}, \bar{y})$ is a quantifier-free $\mathcal{Q}$-formula. If $\mathcal{P} \supseteq \mathcal{N}$ is e.c., the existential sentence $\psi$ still holds in $\mathcal{P} . \exists \bar{y} \varphi(\bar{x}, \bar{y})$ is $T_{1, \ldots, 6}$-equivalent to a quantifierfree formula $\theta(\bar{x})$ of $\mathcal{Q}_{\omega_{1} \omega}$; so $\psi$ is equivalent to $\theta(\bar{m})$ in both $\mathcal{M}$ and $\mathcal{P}$. Because $\mathcal{P} \models \psi, \mathcal{P} \models \theta(\bar{m})$; because $\theta(\bar{m})$ is quantifier-free, $\mathcal{M} \models \theta(\bar{m})$; so $\mathcal{M} \models \psi$ and $\mathcal{M}$ is existentially closed. 


\section{Amalgamation}

A class $\mathcal{S}$ of structures for a language $\mathcal{M}$ has the amalgamation property just in case for all $\mathcal{A}, \mathcal{B}, \mathcal{C} \in \mathcal{S}$ with $\mathcal{A} \subseteq \mathcal{B}, \mathcal{C}$, there are $\mathcal{D} \in \mathcal{S}$, with $\mathcal{B} \subseteq \mathcal{D}$, and an embedding $f: \mathcal{C} \rightarrow \mathcal{D}$ that is the identity on $A$. $T_{1, \ldots, 6}$ is so close to eliminating quantifiers that one may prove

Theorem 4.1. The class of dimension groups has the amalgamation property.

Proof. Let $\mathcal{A} \subseteq \mathcal{B}, \mathcal{C}$ be dimension groups, viewed as $\mathcal{L}$-structures. By taking divisible hulls one may assume that $\mathcal{A}, \mathcal{B}, \mathcal{C}$ are divisible and $\mathcal{A} \subseteq \mathcal{B}, \mathcal{C}$ as $\mathcal{Q}$-structures. By extending $\mathcal{B}, \mathcal{C}$ if necessary one may assume that they are existentially closed. Let

$$
\Gamma=T^{\mathcal{Q}} \cup \operatorname{diag}(\mathcal{B}) \cup \operatorname{diag}(\mathcal{C}),
$$

where $\mathcal{Q}_{B} \cap \mathcal{Q}_{C}=\mathcal{Q}_{A}$. If $\mathcal{D} \models \Gamma$, then one may assume that $\mathcal{B} \subseteq \mathcal{D}\lceil\mathcal{Q}$, and the natural embedding of $\mathcal{C}$ into $\mathcal{D} \backslash \mathcal{Q}$ that takes $e^{\mathcal{C}}$ to $e^{\mathcal{D}}$ when $e \in \mathcal{Q}_{C} \backslash \mathcal{Q}$ will solve the amalgamation problem. The compactness theorem will give $\Gamma$ a model if every set $T^{\mathcal{Q}} \cup \operatorname{diag}(\mathcal{B}) \cup\left\{\varphi_{1}(\bar{a}, \bar{c}), \ldots, \varphi_{n}(\bar{a}, \bar{c})\right\}$ has a model, where the $\varphi_{i}(\bar{x}, \bar{y})$ s are $\mathcal{Q}$ formulas, the as come from $A$, the $c$ s come from $C \backslash A$, and each $\varphi_{i}(\bar{a}, \bar{c}) \in \operatorname{diag}(\mathcal{C})$. Since $\mathcal{B}$ and $\mathcal{C}$ are existentially closed, Theorem 3.5 and the results of Section 2 provide a quantifier-free formula $\theta(\bar{x})$ of $\mathcal{Q}_{\omega_{1} \omega}$ that is equivalent to $\exists \bar{y} \bigwedge_{i} \varphi_{i}(\bar{x}, \bar{y})$ in $\mathcal{B}$ and in $\mathcal{C}$. Since $\mathcal{C} \models \exists \bar{y} \bigwedge_{i} \varphi_{i}(\bar{a}, \bar{y}), \mathcal{C} \models \theta(\bar{a})$; since $\theta(\bar{a})$ is quantifier-free and $\mathcal{A} \subseteq \mathcal{C}, \mathcal{B}, \mathcal{B}=\theta(\bar{a})$; so $\mathcal{B} \models \exists \bar{y} \bigwedge_{i} \varphi_{i}(\bar{a}, \bar{y})$ and $\mathcal{B}$ may be expanded to a model of $T^{\mathcal{Q}} \cup \operatorname{diag}(\mathcal{B}) \cup\left\{\varphi_{1}(\bar{a}, \bar{c}), \ldots, \varphi_{n}(\bar{a}, \bar{c})\right\}$.

The particular form of schemas (1)-(6) allows one to show that

Theorem 4.2. If $\mathcal{N}$ is a nontrivial ideal in the e.c. dimension group $\mathcal{M}$, then $\mathcal{N}$ is an e.c. dimension group.

Proof. One shows that $\mathcal{N}=T_{1, \ldots, 6}$.

The ideal $\mathcal{N}$ is a partially ordered Abelian group with directed, isolated order. Because $\mathcal{N}$ is nontrivial, it is strictly directed. Since the witness $w$ in strict interpolation (2) is bounded by the parameters $x, y, z, v, \mathcal{N}$ obeys strict interpolation. Rational multiples of nonnegative elements of $\mathcal{N}$ are bounded by elements of $\mathcal{N}$ and so belong to $\mathcal{N}$; because it is directed, every element of $\mathcal{N}$ is the difference of two nonnegative elements, and so $\mathcal{N}$ is divisible. If

$$
\bigwedge_{\bar{m} \in \mathbb{N}^{n} \backslash\{\overline{0}\}} 0 \not \leq \sum_{i} m_{i} a_{i}
$$

in $\mathcal{N} \subseteq \mathcal{M}$ and $y \in M$ obeys

$$
\bigwedge_{i} a_{i}<y \wedge 0 \nless y,
$$

then there is $b \in N$ with

$$
\bigwedge_{i} a_{i}<b
$$

because $\mathcal{N}$ is strictly directed, and by strict interpolation in $\mathcal{M}$ there is $c \in M$ with

$$
\bigwedge_{i} a_{i}<c \wedge c<b, y
$$


so $c \in N$, a convex subset of $M$, and $0 \nless c$ because $c<y$ and $0 \nless y$. If

$$
\bigwedge_{s} \bigwedge_{i} a_{i}<y_{s} \wedge \bigwedge_{j} y_{s}<b_{j} \wedge y_{s} \mid w_{s}
$$

in $\mathcal{N}$, which is strictly directed, there is $c \in N$ with

$$
\bigwedge_{s}-c<y_{s}<c
$$

so

$$
\bigwedge_{s}-c<y_{s} \wedge \bigwedge_{i} a_{i}<y_{s} \wedge \bigwedge_{j} y_{s}<b_{j} \wedge y_{s}<c \wedge y_{s} \mid w_{s}
$$

and by schema (4) for $\mathcal{M}$ there is $y \in M$ with

$$
-c<y \wedge \bigwedge_{i} a_{i}<y \wedge \bigwedge_{j} y<b_{j} \wedge y<c \wedge \bigwedge_{s} y \mid w_{s} ;
$$

since $c \in N$ and $-c<y<c, y \in N$. Schema (5) is verified for $\mathcal{N}$ in a similar fashion. If an instance of schema (6) has both $a$ s and $b$ s, it is true in $\mathcal{N}$ for the same reason that $\mathcal{N}$ obeys strict interpolation; but if, for example, no $b$ s are present, one may reduce to the previous case because the nontrivial ideal $\mathcal{N}$ contains a strictly positive element which may play the role of $b$.

So

Corollary 4.3. If $\mathcal{M}$ is a nontrivial dimension group, then there is an e.c. dimension group $\mathcal{N} \supseteq \mathcal{M}$ in which $\mathcal{M}$ is cofinal.

Proof. If $\mathcal{P} \supseteq \mathcal{M}$ is e.c. and $\mathcal{N} \subseteq \mathcal{P}$ is the ideal in $\mathcal{P}$ generated by $\mathcal{M}$-i.e., the set of all elements $x-y$ with $0 \leq x \leq z$ and $0 \leq y \leq v$ for some $z, v \in M$-then $\mathcal{M}$ is cofinal in $\mathcal{N}$ and $\mathcal{N}$ is an e.c. dimension group (Theorem 4.2).

The class of nontrivial dimension groups with distinguished order unit-viewed as structures for $\mathcal{L} \cup\{u\}$ or for $\mathcal{L}^{<} \cup\{u\}$-is inductive, though not elementary; so every such $(\mathcal{M}, u)$ may be embedded in another such dimension group $(\mathcal{N}, u)$ that is e.c. in this class. One may show as follows that $\mathcal{N}$ is e.c. among all dimension groups. Let $\varphi$ be an existential $\mathcal{L}_{N}$-sentence true in some dimension group $\mathcal{P} \supseteq \mathcal{N}$. Because $\varphi$ is existential one may assume that $\mathcal{P}$ is e.c., and so the ideal $\mathcal{I}$ of $\mathcal{P}$ generated by $u>0$ is also e.c. (Theorem 4.2). Since $N \subseteq I$ and $\mathcal{I}$ is e.c., $\varphi$ is true in $\mathcal{I}$; so since $(\mathcal{I}, u)$ is a dimension group with order unit, $\varphi$ is true in $(\mathcal{N}, u)$ and in $\mathcal{N}$. One thus establishes the more difficult direction of

Corollary 4.4. The nontrivial dimension groups with distinguished order unit that are e.c. among such groups are exactly the models of $T_{1, \ldots, 6} \cup\left\{u>0, \forall x \bigvee_{n \geq 1} x \leq\right.$ $n u\}$.

One may also show that

Corollary 4.5. The class of dimension groups with distinguished order unit has the amalgamation property.

Proof. Given three such groups $(\mathcal{A}, u) \subseteq(\mathcal{B}, u),(\mathcal{C}, u)$, invoke Theorem 4.1 to find a dimension group $\mathcal{D} \supseteq \mathcal{B}$ and an embedding $f: \mathcal{C} \rightarrow \mathcal{D}$ that is the identity on $A$. If $\mathcal{E} \subseteq \mathcal{D}$ is the ideal of $\mathcal{D}$ generated by $u$, then $\mathcal{E} \supseteq \mathcal{B}$ is a dimension group containing $\operatorname{ran}(f)$, and so $(\mathcal{E}, u)$ and $f$ solve the given amalgamation problem. 
Rewriting the definition in ([4], p. 7), one says that an element $a$ of a dimension group is a pseudo-zero just in case

$$
a \neq 0 \wedge \forall y(y>0 \leftrightarrow y>a)
$$

For example, if one partially orders the vector space $\mathbb{Q} \times \mathbb{Q}$ so that

$$
(0,0)<(z, v) \text { iff } 0<z \text { in } \mathbb{Q},
$$

then $\mathbb{Q} \times \mathbb{Q}$ becomes a simple dimension group in which $(1,0)$ is an order unit and $(0,1)$ is a pseudo-zero. Since the definition of pseudo-zero is universal, an element $c$ from a dimension group $\mathcal{A}$ will be a pseudo-zero in $\mathcal{A}$ if $c$ is a pseudo-zero in a dimension group $\mathcal{B} \supseteq \mathcal{A}$. The converse may fail because an e.c. dimension group has isolated order and obeys schema (3), and these properties rule out pseudo-zeros. But the converse does hold in the following special case:

Lemma 4.6. If the $\mathcal{Q} \cup\{u\}$-structures $\mathcal{A} \subseteq \mathcal{B}$ are simple divisible partially ordered Abelian groups with isolated order and distinguished order unit, then any pseudozero in $\mathcal{A}$ remains a pseudo-zero in $\mathcal{B}$.

Proof. Let $c \in A$ be a pseudo-zero in $\mathcal{A}$ : so $c \neq 0$ and

$$
\mathcal{A} \models \forall y(y>0 \leftrightarrow y>c)
$$

If $d>0$ in $\mathcal{B}$ - which is simple and divisible and has isolated order - then there is a positive rational $r$ with $d>r u$, and since $r u>0$ in $\mathcal{A}, r u>c$ by hypothesis and $d>c$. Conversely, if $d>c$ in $\mathcal{B}$, then $d-c>0, d-c>s u$ for some positive rational $s$, and $c+s u>c$ in $\mathcal{A}$; so by hypothesis $c+s u>0$ and $d>c+s u>0$. Thus $c$ is a pseudo-zero in $\mathcal{B}$.

So while Corollary 4.3 provides an e.c. dimension group $\mathcal{N} \supseteq \mathbb{Q} \times \mathbb{Q}$ with order unit $(1,0), \mathcal{N}$ cannot be simple.

Pseudo-zeros figure importantly in the proof of

Theorem 4.7. The class of simple dimension groups with distinguished order unit has the amalgamation property.

Proof. Let $\mathcal{A} \subseteq \mathcal{B}, \mathcal{C}$ be simple dimension groups with distinguished order unit $u$. If $\mathcal{A}$ is trivial, then $\mathcal{B}$ and $\mathcal{C}$ are also trivial because all three groups have the same order unit; so one may assume that all the groups are nontrivial. By taking divisible hulls one may assume that $\mathcal{A} \subseteq \mathcal{B}, \mathcal{C}$ as $\mathcal{Q} \cup\{u\}$-structures.

Let $P_{\mathcal{A}}$ be the union of $\{0\}$ with the set of pseudo-zeros of $\mathcal{A}$. By $\left([4,2.2) P_{\mathcal{A}}\right.$ is a trivially ordered 12 subspace of $\mathcal{A}$ and $\mathcal{A} \cong\left(\mathcal{A} / P_{\mathcal{A}}\right) \times P_{\mathcal{A}}$, where $\mathcal{A} / P_{\mathcal{A}}$ carries the usual quotient partial ordering and $\left(\mathcal{A} / P_{\mathcal{A}}\right) \times P_{\mathcal{A}}$ is partially ordered so that

$$
(0,0)<(z, v) \text { iff } 0<z .
$$

([4], 2.2) shows that $\mathcal{A} / P_{\mathcal{A}}$ has no pseudo-zeros, and one easily verifies that $\mathcal{A} / P_{\mathcal{A}}$ is a simple divisible dimension group. If one defines $P_{\mathcal{B}}$ and $P_{\mathcal{C}}$ in a similar fashion, one reaches similar conclusions about $\mathcal{B} \cong\left(\mathcal{B} / P_{\mathcal{B}}\right) \times P_{\mathcal{B}}$ and $\mathcal{C} \cong\left(\mathcal{C} / P_{\mathcal{C}}\right) \times P_{\mathcal{C}}$. Lemma 4.6. says that

$$
P_{\mathcal{A}}=A \cap P_{\mathcal{B}}=A \cap P_{\mathcal{C}}
$$

Therefore, $P_{\mathcal{A}} \subseteq P_{\mathcal{B}}, P_{\mathcal{C}}$ as trivially ordered vector spaces and $\mathcal{A} / P_{\mathcal{A}} \subseteq \mathcal{B} / P_{\mathcal{B}}, \mathcal{C} / P_{\mathcal{C}}$ as $\mathcal{Q} \cup\{u\}$-structures. One may certainly find a trivially ordered vector space $V \supseteq P_{\mathcal{B}}$ and an injective linear map $f_{0}: P_{\mathcal{C}} \rightarrow V$ with $f_{0} \uparrow P_{\mathcal{A}}$ the identity on

\footnotetext{
${ }^{12}$ I.e., 0 is the only nonnegative element.
} 
$P_{\mathcal{A}}$. Suppose one can find a simple divisible dimension group $\mathcal{M} \supseteq \mathcal{B} / P_{\mathcal{B}}$ and a $\mathcal{Q} \cup\{u\}$-embedding $f_{1}: \mathcal{C} / P_{\mathcal{C}} \rightarrow \mathcal{M}$ such that $f_{1}$ is the identity on $\mathcal{A} / P_{\mathcal{A}}$ and $\mathcal{M}$ satisfies strict interpolation. Partially ordering $\mathcal{M} \times V$ so that

$$
(0,0)<(m, v) \text { iff } 0<m,
$$

one creates a divisible partially ordered Abelian group with directed, isolated order, and because $\mathcal{M}$ satisfies strict interpolation so does $\mathcal{M} \times V . \mathcal{M} \times V \supseteq\left(\mathcal{B} / P_{\mathcal{B}}\right) \times P_{\mathcal{B}}$ will be simple, and together with $\left(f_{0}, f_{1}\right):\left(\mathcal{C} / P_{\mathcal{C}}\right) \times P_{\mathcal{C}} \rightarrow \mathcal{M} \times V$ will solve the amalgamation problem for

$$
\left(\mathcal{A} / P_{\mathcal{A}}\right) \times P_{\mathcal{A}} \subseteq\left(\mathcal{B} / P_{\mathcal{B}}\right) \times P_{\mathcal{B}},\left(\mathcal{C} / P_{\mathcal{C}}\right) \times P_{\mathcal{C}} ;
$$

exploiting the isomorphisms between these groups and $\mathcal{A} \subseteq \mathcal{B}, \mathcal{C}$, one solves the original amalgamation problem.

The discussion so far has reduced the solution of the problem to the following task: given $\mathcal{Q} \cup\{u\}$-structures $\mathcal{A} \subseteq \mathcal{B}, \mathcal{C}$ that are nontrivial simple divisible dimension groups without pseudo-zeros, find a simple divisible dimension group $\mathcal{M} \supseteq \mathcal{B}$, satisfying strict interpolation, and a $\mathcal{Q} \cup\{u\}$-embedding $h: \mathcal{C} \rightarrow \mathcal{M}$ that is the identity on $A$. Corollary 4.5 provides a divisible dimension group $\mathcal{D} \supseteq \mathcal{B}$, with order unit $u$, and a $\mathcal{Q} \cup\{u\}$-embedding $f: \mathcal{C} \rightarrow \mathcal{D}$ that is the identity on $A$. The rest of the argument will collapse $\mathcal{D}$ to a simple dimension group without disturbing the copies of $\mathcal{B}$ and $\mathcal{C}$ inside $\mathcal{D}$, and borrows heavily from the proof of $(4,15.1)$, a representation theorem for a special class of simple dimension groups.

Let $\mathcal{G}$ be the set of maximal proper convex subgroups of $\mathcal{D}$. Because $\mathcal{D}$ has an order unit and is nontrivial, $\mathcal{G} \neq \emptyset$, and since every $K \in \mathcal{G}$ is maximal, every such $K$ is also a $\mathbb{Q}$-subspace of $\mathcal{D}$. If $K \in \mathcal{G}$, the nontrivial partially ordered vector space $\mathcal{G} / K$ has isolated order and an order unit but lacks nontrivial convex subgroups, and so may be identified with a unique ordered subgroup of $\mathbb{R}$ (with $1 \in \mathbb{R}$ corresponding to $u+K \in \mathcal{G} / K)$. Let $\varphi_{K}: \mathcal{D} \rightarrow \mathbb{R}$ be the corresponding $\mathbb{Q}$-linear $\mathcal{L} \cup\{u\}$ homomorphism and $\mathcal{H}$ be the set of all $\mathbb{Q}$-linear $\mathcal{L} \cup\{u\}$-homomorphisms of $\mathcal{D}$ into $\mathbb{R}$. For all $K \in \mathcal{G}$ and $\psi \in \mathcal{H}, \varphi_{K} \in \mathcal{H}$ and $\operatorname{ker}(\psi) \in \mathcal{G}$.

Impose the product topology on $\mathbb{R}^{D}$, the set of all real-valued functions with domain $D$. For $a, b, c \in D$ and $q \in \mathbb{Q}$ let

$$
S_{a, b, c}=\left\{\psi \in \mathbb{R}^{D}: \psi(a)+\psi(b)=\psi(c)\right\}
$$

when $a+b=c$,

$$
P_{a, b}=\left\{\psi \in \mathbb{R}^{D}: q \psi(a)=\psi(b)\right\}
$$

when $q a=b$,

$$
G_{a}=\left\{\psi \in \mathbb{R}^{D}: \psi(a) \geq 0\right\}
$$

when $a \geq 0$, and

$$
U=\left\{\psi \in \mathbb{R}^{D}: \psi(u)=1\right\} .
$$

All these sets are closed in $\mathbb{R}^{D}$, as is their intersection $\mathcal{H}$. One may see that $\mathcal{H}$ is compact by looking at the restrictions $\psi \uparrow[-u, u]$ of elements $\psi$ of $\mathcal{H}$ to $[-u, u]=\{d \in D:-u \leq d \leq u\}$. The mapping $R: \mathcal{H} \rightarrow[-1,1]^{[-u, u]}$ sending each $\psi \in \mathcal{H}$ to $\psi \uparrow[-u, u]$ is one-to-one, since if $d \in D,-u \leq r d \leq u$ for some positive rational $r$ and $\psi(d)=(1 / r) R(\psi)(r d)$. If one imposes the product topology on $[-1,1]^{[-u, u]}$ it is compact. For $a, b, c \in[-u, u]$ and $q \in \mathbb{Q}$ let

$$
S_{a, b, c}^{\prime}=\left\{\psi \in[-1,1]^{[-u, u]}: \psi(a)+\psi(b)=\psi(c)\right\}
$$


when $a+b=c$,

$$
P_{a, b}^{\prime}=\left\{\psi \in[-1,1]^{[-u, u]}: q \psi(a)=\psi(b)\right\}
$$

when $q a=b$,

$$
G_{a}^{\prime}=\left\{\psi \in[-1,1]^{[-u, u]}: \psi(a) \geq 0\right\}
$$

when $a \geq 0$, and

$$
U^{\prime}=\left\{\psi \in[-1,1]^{[-u, u]}: \psi(u)=1\right\} .
$$

All these sets are closed in $[-1,1]^{[-u, u]}$, as is their intersection $I \supseteq \operatorname{ran}(R)$. One may show as follows that $I=\operatorname{ran}(R)$. If $\theta \in I$ and $d \in D$, there is a positive rational $r$ with $-u \leq r d \leq u$, and $(1 / r) \theta(r d)$ is defined. If $s$ is another positive rational with $-u \leq s d \leq u$, then $r d, s d \in[-u, u]$ obey $r d=(r / s) s d$,

$$
\theta(r d)=(r / s) \theta(s d)
$$

since $\theta \in I$, and $(1 / r) \theta(r d)=(1 / s) \theta(s d)$. One may therefore define $\bar{\theta}: D \rightarrow \mathbb{R}$ by

$$
\bar{\theta}(d)=(1 / r) \theta(r d) \text { when } 0<r \in \mathbb{Q} \text { and }-u \leq r d \leq u
$$

and show that $\bar{\theta} \in \mathcal{H}$ and $R(\bar{\theta})=\theta$. If $d_{1}, \ldots, d_{n} \in D, s_{1}, \ldots, s_{n}$ are reals, $\epsilon_{1}, \ldots, \epsilon_{n}$ are positive reals, and $r_{1}, \ldots, r_{n}$ are positive rationals with

$$
\bigwedge_{i=1}^{n}-u \leq r_{i} d_{i} \leq u
$$

then for all $\psi \in \mathcal{H}$

$$
\begin{aligned}
\bigwedge_{i=1}^{n}\left|\psi\left(d_{i}\right)-s_{i}\right|<\epsilon_{i} & \text { iff } \\
& \bigwedge_{i=1}^{n}\left|\psi\left(r_{i} d_{i}\right)-r_{i} s_{i}\right|<r_{i} \epsilon_{i} \\
& \text { iff } \bigwedge_{i=1}^{n}\left|R(\psi)\left(r_{i} d_{i}\right)-r_{i} s_{i}\right|<r_{i} \epsilon_{i} .
\end{aligned}
$$

One concludes that $R^{-1}: I \rightarrow \mathcal{H}$ is a continuous bijection; since $I$ is compact, $R^{-1}$ is a homeomorphism and $\mathcal{H}$ is compact.

For $d \in D$ let $\varphi(d): \mathcal{H} \rightarrow \mathbb{R}$ be given for $\psi \in \mathcal{H}$ by

$$
\varphi(d)(\psi)=\psi(d) .
$$

If $\psi \in \mathcal{H}$ and $\epsilon>0$ in $\mathbb{R}$, then $U=\{\gamma \in \mathcal{H}:|\gamma(d)-\psi(d)|<\epsilon\}$ is an open neighborhood of $\psi$ with

$$
\varphi(d)(U) \subseteq(\psi(d)-\epsilon, \psi(d)+\epsilon)=(\varphi(d)(\psi)-\epsilon, \varphi(d)(\psi)+\epsilon) ;
$$

so $\varphi(d) \in C(\mathcal{H})$, the group of continuous real-valued functions on $\mathcal{H}$. If one partially orders $C(\mathcal{H})$ so that

$$
h<k \text { iff } h(\psi)<k(\psi) \text { for all } \psi \in \mathcal{H},
$$

then $C(\mathcal{H})$ is a simple divisible dimension group, with order-unit $\mathcal{H} \rightarrow 1$, that satisfies strict interpolation, and $\varphi: \mathcal{D} \rightarrow C(\mathcal{H})$ is a $\mathbb{Q}$-linear map that sends $u$ to $\mathcal{H} \rightarrow 1$.

$\operatorname{ker}(\varphi)=\bigcap_{\psi \in \mathcal{H}} \operatorname{ker}(\psi)=\bigcap_{H \in \mathcal{G}} H$, so if $b \in B \cap \operatorname{ker}(\varphi), b \in \bigcap_{H \in \mathcal{G}} B \cap H$. Because $\mathcal{B}$ is simple, the maximal convex subgroups of $\mathcal{B}$ are the maximal subgroups consisting of pairwise incomparable elements, and every such subgroup is by Zorn's Lemma contained in a maximal proper convex subgroup of $\mathcal{D}$. So every $b \in B \cap$ $\operatorname{ker}(\varphi)$ belongs to every maximal subgroup of pairwise incomparable elements of $\mathcal{B}$. 
Since $(\llbracket, 2.3)$ shows that such $b$ s are pseudo-zeros if nonzero, $B \cap \operatorname{ker}(\varphi)=\{0\}$ by hypothesis and $\varphi\lceil B$ is injective. Similarly, $\varphi \circ f$ is injective.

If $0<b \in B$, then since $\mathcal{B}$ is simple and divisible there is a positive rational $r$ with $r u \leq b$, and so for every $\psi \in \mathcal{H}$,

$$
\varphi(b)(\psi)=\psi(b) \geq \psi(r u)=r>0
$$

and $\varphi(b)>0$ in $C(\mathcal{H})$. Conversely, if $\varphi(b)>0$, then certainly $b \neq 0$, and from what has just been shown, $b \nless 0$. If $b \ngtr 0$, then $b \mid 0, \mathbb{Q} \cdot b$ is a subspace of $\mathcal{B}$ consisting of pairwise incomparable elements, and there is $K \in \mathcal{G}$ with $\mathbb{Q} \cdot b \subseteq K$; so

$$
\varphi(b)\left(\varphi_{K}\right)=\varphi_{K}(b)=0
$$

and $\varphi(b) \ngtr 0$. This contradiction shows that $b>0$ if $\varphi(b)>0$, and so $\varphi\lceil B$ is a $\mathcal{Q} \cup\{u\}$-embedding of $\mathcal{B}$ into $C(\mathcal{H})$. Similarly, $\varphi \circ f$ is a $\mathcal{Q} \cup\{u\}$-embedding of $\mathcal{C}$ into $C(\mathcal{H})$.

Because $f\lceil A$ is the identity on $A \subseteq B,(\varphi \circ f) \uparrow A=(\varphi\lceil B) \uparrow A$. Therefore the amalgamation problem is solved.

The proofs of Theorem 4.1, Corollary 4.5, and Theorem 4.7 continue to work when $\mathcal{A}$ is any common substructure of $\mathcal{B}$ and $\mathcal{C}$ in the appropriate language: $\mathcal{L}$ for Theorem 4.1 and $\mathcal{L} \cup\{u\}$ for Corollary 4.5 and Theorem 4.7. So in Theorem 4.1, for example, $\mathcal{A}$ may be a substructure of a dimension group rather than a dimension group.

\section{ISOLATED QUANTIFIER-FREE TYPES}

As in ([5], p. 87), a (maximal) quantifier-free $n$-type is a maximal set of quantifier-free $\mathcal{Q}$-formulas, in $n$ distinct variables $x_{1}, \ldots, x_{n}$, consistent with $T^{\mathcal{Q}}$ (or with $T_{1, \ldots, 6}$, since every model of $T^{\mathcal{Q}}$ has an e.c. extension). If $\Gamma\left(x_{1}, \ldots, x_{n}\right)$ is a quantifier-free $n$-type and $\psi\left(x_{1}, \ldots, x_{n}\right)$ is an existential $\mathcal{Q}$-formula, $\psi$ is said to isolate $\Gamma$ just in case $T^{\mathcal{Q}} \cup\{\exists \bar{x} \psi\}$ is consistent and $\psi$ implies, modulo $T^{\mathcal{Q}}$, every formula in $\Gamma$. This section will describe the quantifier-free $n$-types that may be isolated by existential formulas, and conclude that when $n>1$ every e.c. dimension group realizes a nonisolated $n$-type.

A quantifier-free 1-type in $x$ must determine whether $x$ is positive, negative, zero, or incomparable with zero, but this information determines the quantifier-free type. Thus

Theorem 5.1. The quantifier-free 1-types in $x$ are those isolated by the formulas $x>0, x<0, x=0$, and $x \mid 0$.

Suppose $n>1$ and $\Gamma$ is a quantifier-free $n$-type in $x_{1}, \ldots, x_{n}$. The definitions imply that

Lemma 5.2. Suppose $\Gamma$ contains a nontrivial identity, i.e. a formula

$$
b_{1} x_{1}+\cdots+b_{n} x_{n}=0
$$

in which some $b_{i} \in \mathbb{Q} \backslash\{0\}$. Let $\Gamma^{\prime}$ be the quantifier-free $(n-1)$-type consisting of all formulas in $\Gamma$ that do not contain $x_{i}$. If $\psi\left(x_{1}, \ldots, x_{i-1}, x_{i+1}, \ldots, x_{n}\right)$ is an existential $\mathcal{Q}$-formula, $\psi$ isolates $\Gamma^{\prime}$ just in case $\psi \wedge b_{1} x_{1}+\cdots+b_{n} x_{n}=0$ isolates $\Gamma$.

So from now on one may assume that $\Gamma$ contains no nontrivial identities. 
Lemma 5.3. If $\Gamma$ contains $c \mid 0$ whenever $c$ is a nonzero $\mathbb{Q}$-linear form, then $\Gamma$ is isolated.

Proof. These formulas $c \mid 0$ determine $\Gamma$; so if there is an existential $\mathcal{Q}$-formula $T_{1, \ldots, 6^{-}}$ equivalent to

$$
\bigwedge_{\bar{c} \in \mathbb{Q}^{n} \backslash\{\overline{0}\}} \sum_{i} c_{i} x_{i} \mid 0
$$

that formula will isolate $\Gamma$. The displayed formula is $T^{\mathcal{Q}}$-equivalent to

$$
\bigwedge_{\bar{m} \in \mathbb{Z}^{n} \backslash\{\overline{0}\}} 0 \not \leq \sum_{i} m_{i} x_{i}
$$

and to

$$
\bigwedge_{j:\{1, \ldots, n\} \rightarrow\{0,1\}} \bigwedge_{\bar{m} \in \mathbb{N}^{n} \backslash\{\overline{0}\}} 0 \not \leq \sum_{i} m_{i}(-1)^{j(i)} x_{i} .
$$

Schema (3) makes this formula $T_{1, \ldots, 6}$-equivalent to

$$
\bigwedge_{j:\{1, \ldots, n\} \rightarrow\{0,1\}} \exists y\left(\bigwedge_{i=1}^{n}(-1)^{j(i)} x_{i}<y \wedge 0 \nless y\right) .
$$

If one assumes now that $\Gamma$ contains no nontrivial identities and contains $c \nmid 0$ for some nonzero $\mathbb{Q}$-linear form $c$, then $\Gamma$ must contain $a>0$ for some nonzero $\mathbb{Q}$-linear form $a$. To treat this case one needs

Lemma 5.4. Any existential $\mathcal{Q}$-sentence consistent with $T^{\mathcal{Q}}$ may be satisfied in the $\mathcal{Q}$-power $\mathbb{Q}^{n}$ for some positive integer $n$.

Proof. Suppose that the existential sentence $\psi$ is true in the divisible dimension group $\mathcal{M}$. If $M=\{0\}$ there is nothing to prove; so assume $M \neq\{0\}$. As in Section $2, \mathcal{M}$ may be embedded in the dimension group

$$
\widehat{\mathcal{M}}=\prod_{\prec \in \mathcal{O}(\mathcal{M})}(M, \prec)
$$

by the diagonal map $\delta: M \rightarrow \widehat{M}$, and each $(M, \prec)$ is a nontrivial divisible ordered Abelian group. Since the negation of an atomic condition is satisfied in a product just in case the atomic condition fails in at least one coordinate, $\psi$ may be satisfied in a finite product of nontrivial divisible ordered Abelian groups. The FefermanVaught theorem makes this product elementarily equivalent to a finite power of $\mathbb{Q}$, and so the desired result holds.

Returning to the quantifier-free $n$-type $\Gamma$ with $n>1$, one may state

Theorem 5.5. If $\Gamma$ contains an inequality but no nontrivial identities, then $\Gamma$ is not isolated.

Proof. Suppose, on the contrary, that the existential $\mathcal{Q}$-formula $\psi\left(x_{1}, \ldots, x_{n}\right)$ isolates $\Gamma$. Lemmas 3.13 .4 make $\psi T_{1,2,4,5,6}$-equivalent to a disjunction of formulas

$$
\bigwedge_{i} a_{i}>0 \wedge \bigwedge_{j} b_{j}=0 \wedge \bigwedge_{k} c_{k} \mid 0 \wedge \bigwedge_{l} \exists z_{l}\left(\bigwedge_{m} d_{l m}<z_{l} \wedge 0 \nless z_{l}\right)
$$

in which the $a \mathrm{~s}, b \mathrm{~s}, c \mathrm{~s}$, and $d \mathrm{~s}$ are terms in $\bar{x}$ and the $z \mathrm{~s}$ occur only as shown. Because any disjunct consistent with $T_{1, \ldots, 6}$ will also isolate $\Gamma$, one may assume that $\psi$ is the displayed formula. Since $\Gamma$ contains no nontrivial identities, one may assume that 
there are no conjuncts $b_{j}=0$; because $\Gamma$ contains at least one inequality (and is consistent with $T_{1, \ldots, 6}$ ), one may assume that there is at least one conjunct $a_{i}>0$.

In each such conjunct $a_{i}$ is a $\mathbb{Q}$-linear form $h_{i} \cdot \bar{x}$ with $h_{i} \in \mathbb{Q}^{n} \backslash\{\overline{0}\}$. Let $C \subseteq \mathbb{Q}^{n}$ be the cone generated by $h_{1}, \ldots, h_{n}$ : i.e.,

$$
C=\left\{\sum_{i} r_{i} h_{i}: 0 \leq r_{i} \in \mathbb{Q} \text { for } i=1, \ldots,|I|\right\} .
$$

Since $T^{\mathcal{Q}} \cup\left\{\exists \bar{x} \bigwedge_{i} a_{i}>0\right\}$ is consistent, $\{\overline{0}\}$ is the only subspace of $\mathbb{Q}^{|I|}$ contained in $C$. By removing redundant inequalities one may assume that no proper subset of $\left\{h_{1}, \ldots, h_{|I|}\right\}$ generates $C$ : i.e., for all $J \subset\{1, \ldots,|I|\}$,

$$
C \neq\left\{\sum_{i \in J} r_{i} h_{i}: 0 \leq r_{i} \in \mathbb{Q} \text { for all } i \in J\right\} .
$$

Thus no $h_{i}$ is a linear combination, by nonnegative scalars, of the $h_{j}$ with $j \neq i$.

Suppose first that $|I|>1$. Because $\exists \bar{x} \psi(\bar{x})$ is consistent with $T^{\mathcal{Q}}$, Lemma 5.4 implies that

$$
\bigwedge_{i} a_{i}>0 \wedge \bigwedge_{k} c_{k} \mid 0 \wedge \bigwedge_{l} \bigwedge_{m} d_{l m}<z_{l} \wedge 0 \nless z_{l}
$$

may be satisfied in the $\mathcal{Q}$-power $\mathbb{Q}^{s}$ for some positive integer $s$. Since $a_{2}>0$ in the simple divisible dimension group $\mathbb{Q}^{s}$, there is a positive rational $r$ with

$$
a_{2}>r a_{1}
$$

in $\mathbb{Q}^{s}$.

To argue by contradiction that $a_{2}>r a_{1}$ is independent of $\psi$, suppose that

$$
\mathbb{Q} \models \forall \bar{x}, \bar{z}\left(\bigwedge_{i} a_{i}>0 \wedge \bigwedge_{l, m} d_{l m}<z_{l} \rightarrow a_{2}>r a_{1}\right) .
$$

Whenever every $a_{i}>0$, one may choose the $z$ s sufficiently large so that every $d_{l m}<z_{l}$, and then $a_{2}>r a_{1}$; so

$$
\mathbb{Q} \models \forall \bar{x}\left(\bigwedge_{i} a_{i}>0 \rightarrow a_{2}>r a_{1}\right) .
$$

Assume, temporarily,

Lemma 5.6. Let $F$ be an ordered field, $A$ a $k \times l$ matrix over $F$, and $b \in F^{k}$. Suppose $\exists y\left(y^{T} A>0\right) 13$ The following conditions are equivalent:

(i) $b=A z$ for some $z \in F^{l}$ with $z \geq 0$ and $\sum_{i} z_{i}>0$.

(ii) $\forall y\left(y^{T} A>0 \rightarrow y^{T} b>0\right)$.

Because

$$
\mathbb{Q} \models \forall x\left(x^{\mathrm{T}}\left(h_{1} \ldots h_{|I|}\right)>0 \rightarrow x^{\mathrm{T}}\left(h_{2}-r h_{1}\right)>0\right),
$$

Lemma 5.6 provides $t \geq 0$ in $\mathbb{Q}^{|I|}$ with $\sum_{i} t_{i}>0$ and

$$
\begin{gathered}
h_{2}-r h_{1}=\sum_{i} t_{i} h_{i}, \\
\therefore\left(1-t_{2}\right) h_{2}=\left(r+t_{1}\right) h_{1}+\sum_{i>2} t_{i} h_{i} .
\end{gathered}
$$

\footnotetext{
${ }^{13}$ The row vector $y^{T}$ is the transpose of the column vector $y$, and $\geq 0(>0)$ means nonnegative (positive) in every coordinate.
} 
If $1-t_{2}>0$, then $h_{2}$ is a linear combination, by nonnegative scalars, of the $h_{j}$ with $j \neq 2$; if $1-t_{2}=0$, then $-h_{1} \in C$, which contains the subspace $\mathbb{Q} h_{1} \neq\{\overline{0}\}$ of $\mathbb{Q}^{|I|}$; if $1-t_{2}<0$, then $-h_{2} \in C$, which contains the subspace $\mathbb{Q} h_{2} \neq\{\overline{0}\}$ of $\mathbb{Q}^{|I|}$. This contradiction implies that

$$
\mathbb{Q} \models \exists \bar{x}, \bar{z}\left(\bigwedge_{i} a_{i}>0 \wedge \bigwedge_{l, m} d_{l m}<z_{l} \wedge a_{2} \ngtr r a_{1}\right) .
$$

Expanding the previous interpretation in $\mathbb{Q}^{s}$ to one in $\mathbb{Q}^{s+1}$ in which $\bar{x}$ and $\bar{z}$ receive, in coordinates $s+1$, values corresponding to the last display, one obtains an interpretation making $\psi \wedge a_{2} \ngtr r a_{1}$ true. So modulo $T^{\mathcal{Q}}, \psi$ is consistent with $a_{2}>r a_{1}$ and also with $a_{2} \ngtr r a_{1}$, contrary to the assumption that $\psi$ isolates $\Gamma$.

Assume now that $|I|=1$. Since $a_{1}$ is a nonzero linear form in $\bar{x}$ and $n>1$, there is a variable $x_{i}$ such that $a_{1}$ is not a multiple of $x_{i}$. As before, the conjunction

$$
a_{1}>0 \wedge \bigwedge_{k} c_{k} \mid 0 \wedge \bigwedge_{l} \bigwedge_{m} d_{l m}<z_{l} \wedge 0 \nless z_{l}
$$

may be satisfied in the $\mathcal{Q}$-product $\mathbb{Q}^{s}$ for some positive integer $s . a_{1}$ becomes an order unit under this interpretation, and so there is $r>0$ in $\mathbb{Q}$ such that

$$
a_{1}-r x_{i}>0
$$

in $\mathbb{Q}^{s}$. Since $T^{\mathcal{Q}} \cup\left\{\psi, a_{1}-r x_{i}>0\right\}$ is satisfiable and $\psi$ isolates $\Gamma, a_{1}-r x_{i}>0$ belongs to $\Gamma$ and $\psi \wedge a_{1}-r x_{i}>0$ also isolates $\Gamma$. Because $a_{1}$ and $a_{1}-r x_{i}$ are linearly independent linear forms, $\psi \wedge a_{1}-r x_{i}>0$ falls victim to the analysis given when $|I|>1$, and one again reaches a contradiction.

So the argument for Theorem 5.5 will be complete when Lemma 5.6 is established. Certainly (i) implies (ii): for if $b=A z$ for some $z \in F^{l}$ with $z \geq 0$ and $\sum_{i} z_{i}>0$, then whenever $y^{\mathrm{T}} A>0$,

$$
y^{\mathrm{T}} b=y^{\mathrm{T}}(A z)=\left(y^{\mathrm{T}} A\right) z>0
$$

since $y^{\mathrm{T}} A, z \geq 0$, every coordinate of $y^{\mathrm{T}} A$ is positive, and some coordinate of $z$ is positive.

Now assume that $\exists y\left(y^{\mathrm{T}} A>0\right)$ and that (ii) holds. If

$$
\forall w>0 \exists y\left(y^{\mathrm{T}} A \geq\left(\begin{array}{c}
1 \\
\vdots \\
1
\end{array}\right) \wedge y^{\mathrm{T}} b<w\right)
$$

in $F$, then there is an $F$-semilinear $g: F \rightarrow F^{k}$ with

$$
\forall w>0\left(g(w)^{\mathrm{T}} A \geq\left(\begin{array}{c}
1 \\
\vdots \\
1
\end{array}\right) \wedge g(w)^{\mathrm{T}} b<w\right)
$$

in $F 14$ Because $g_{0}=\lim _{w \rightarrow 0^{+}} g(w)$ exists in $F^{k}$,

$$
g_{0}^{\mathrm{T}} A \geq\left(\begin{array}{c}
1 \\
\vdots \\
1
\end{array}\right) \wedge g_{0}^{\mathrm{T}} b \leq 0,
$$

\footnotetext{
${ }^{14}$ The notions and results, exploited here, about semilinear sets may be found in the Appendix to 8 .
} 
contrary to hypothesis. Thus there is $h>0$ for which

$$
\forall y\left(y^{\mathrm{T}} A \geq\left(\begin{array}{c}
1 \\
\vdots \\
1
\end{array}\right) \rightarrow y^{\mathrm{T}} b \geq h\right)
$$

and

$$
\forall y, z\left(y^{\mathrm{T}} A \geq\left(\begin{array}{c}
z \\
\vdots \\
z
\end{array}\right)>0 \rightarrow y^{\mathrm{T}} b \geq h z\right)
$$

in $F$. If there is $y_{0}$ with

$$
y_{0}^{\mathrm{T}} A \geq 0 \wedge y_{0}^{\mathrm{T}} b<0,
$$

and one picks $y_{1}$ with $y_{1}^{\mathrm{T}} A>0$, then for all positive $r \in F$

$$
\left(y_{1}+r y_{0}\right)^{\mathrm{T}} A=y_{1}^{\mathrm{T}} A+r\left(y_{0}^{\mathrm{T}} A\right)>0
$$

and by (ii)

$$
\left(y_{1}+r y_{0}\right)^{\mathrm{T}} b=y_{1}^{\mathrm{T}} b+r\left(y_{0}^{\mathrm{T}} b\right)>0 ;
$$

but this last inequality must fail for sufficiently large $r \in F$. Thus

$$
\forall y, z\left(y^{\mathrm{T}} A \geq\left(\begin{array}{c}
z \\
\vdots \\
z
\end{array}\right) \geq 0 \rightarrow y^{\mathrm{T}} b \geq h z\right)
$$

and

$$
\forall y, z\left(\left(y^{\mathrm{T}} z\right)\left(\begin{array}{rrrr} 
& & & 0 \\
& A & & \vdots \\
& & & 0 \\
-1 & \ldots & -1 & 1
\end{array}\right) \geq 0 \rightarrow\left(y^{\mathrm{T}} z\right)\left(\begin{array}{c}
b \\
-h
\end{array}\right) \geq 0\right) .
$$

Farkas' Lemma now supplies nonnegative $u \in F^{l}, v \in F$ with

$$
\left(\begin{array}{c}
b \\
-h
\end{array}\right)=\left(\begin{array}{rrrr} 
& & & 0 \\
& A & & \vdots \\
-1 & \ldots & -1 & 1
\end{array}\right)\left(\begin{array}{l}
u \\
v
\end{array}\right)
$$

and so

$$
b=A u,-h=-\sum_{i} u_{i}+v,
$$

and $\sum_{i} u_{i}=h+v \geq h>0$.

One easily concludes, from the analysis of isolated quantifier-free $n$-types, that

Corollary 5.7. If $n>1$, every e.c. dimension group realizes nonisolated quantifier-free $n$-types.

Proof. Let $\mathcal{D}$ be an e.c. dimension group. $\mathcal{D}$ is nontrivial and so has a positive element $e_{1}$; schema (6) now provides an element $e_{2} \mid 0$. $e_{1}$ and $e_{2}$ must be linearly independent; so the quantifier-free type of $\left(e_{1}, e_{2}\right)$ in $\mathcal{D}$ contains an inequality and no nontrivial identities and is not isolated (Theorem 5.5). If $n>2$ and 
$e_{3}, \ldots, e_{n} \in D$, then an existential formula $\psi\left(x_{1}, \ldots, x_{n}\right)$ isolating the quantifierfree type of $\left(e_{1}, \ldots, e_{n}\right)$ in $\mathcal{D}$ would yield an existential formula $\exists x_{3} \ldots \exists x_{n} \psi$ isolating the quantifier-free type of $\left(e_{1}, e_{2}\right)$ in $\mathcal{D}$; so the quantifier-free type of $\left(e_{1}, \ldots, e_{n}\right)$ in $\mathcal{D}$ is not isolated.

\section{Generic Dimension GROUps}

The results of Section 5 have an immediate application to the study of finitely generic dimension groups, i.e., finitely generic models of $T^{\mathcal{Q}}$. According to Theorems 4.1.4 and 4.1.5 and the proof of Theorem 4.1.6 in ([5], pp. 88-91), there is a family $\left\{\mathcal{D}_{\alpha}\right\}_{\alpha<2 \aleph_{0}}$ of countable finitely generic dimension groups with the following property: when $\alpha \neq \beta$, the only quantifier-free types realized in both $\mathcal{D}_{\alpha}$ and $\mathcal{D}_{\beta}$ are isolated. Since finitely generic structures are e.c., every $\mathcal{D}_{\alpha}$ realizes a nonisolated quantifier-free 2-type (Corollary 5.7); so since embeddings preserve quantifier-free types, $\mathcal{D}_{\alpha}$ may not be embedded in $\mathcal{D}_{\beta}$ when $\alpha \neq \beta$. Thus

Corollary 6.1. There is a family of continuum-many countable, finitely generic dimension groups that are pairwise nonembeddable.

Because an algebraically prime e.c. dimension group would realize a nonisolated quantifier-free 2-type that would be realized in every $\mathcal{D}_{\alpha}$, one concludes that

Corollary 6.2. There is no algebraically prime e.c. dimension group.

Despite the complexity revealed by Corollary 6.1, all finitely generic dimension groups are elementarily equivalent because the $\mathcal{L}$-theory of dimension groups has the joint-embedding property (take the usual direct sum). The rest of this section will find properties shared by all finitely generic dimension groups or by all infinitely generic dimension groups; the direct-sum argument again implies that all of the latter are elementarily equivalent.

It will be convenient to consider model-theoretic forcing with respect to certain extensions of $T^{\mathcal{Q}}$. For each $\mathcal{L}$-structure $\mathcal{G}$ which is an at most countable divisible ordered Abelian group, let

$$
T_{\mathcal{G}}^{\mathcal{Q}}=T^{\mathcal{Q}} \cup \operatorname{diag}(\mathcal{G})
$$

$T_{\mathcal{G}}^{\mathcal{Q}}$ is a countable $\forall \exists$-theory, relative to which one may consider the notions of e.c. (finitely generic, infinitely generic). Models in these classes will be called $\mathcal{G}$-e.c. ( $\mathcal{G}$ finitely generic, $\mathcal{G}$-infinitely generic), while e.c. (finitely generic, infinitely generic) will refer to $T^{\mathcal{Q}}=T_{\{0\}}^{\mathcal{Q}}$. In all cases, $W$ will be the countable set of witnesses ([5], p. 27).

The following result generalizes Lemma 5.4.

Lemma 6.3. If $p$ is a condition for finite forcing relative to $T_{\mathcal{G}}^{\mathcal{Q}}$, then $p$ may be satisfied in the $\mathcal{Q}_{\mathcal{G}}$-structure $\mathcal{G}^{n}$ for some positive integer $n\left(\mathbb{Q}^{n}\right.$, if $\left.\mathcal{G}=\{0\}\right)$.

Proof. The argument is essentially that for Lemma 5.4, with $T^{\mathcal{Q}}$ replaced by $T_{\mathcal{G}}^{\mathcal{Q}}$ and $\mathcal{M}$ now a dimension group extending $\mathcal{G}$. Since $\mathcal{G}$ is a linearly ordered Abelian group, the projection of $\delta(G) \subseteq \widehat{M}$ on the coordinate determined by $\prec \in \mathcal{O}(\mathcal{M})$ gives an ordered subgroup of $(M, \prec)$ isomorphic to $\mathcal{G}$; so when $G \neq\{0\}$ one may replace $\mathbb{Q}$ by $\mathcal{G}$ in the previous argument. 
This result implies that

Lemma 6.4. If $\mathcal{G}$ is also Archimedean, then in finite forcing relative to $T_{\mathcal{G}}^{\mathcal{Q}}$,

$$
\{v>0\} \Vdash \forall x \bigvee_{m \geq 1}-m v \leq x \leq m v .
$$

Proof. By Theorem 3.4.4 of [5] one need show merely that if $p \supseteq\{v>0\}$ is a condition and $d \in W$ is not in $p$, then there are a condition $q \supseteq p$ and an integer $m \geq 1$ with $q \Vdash-m v \leq d \leq m v$. Lemma 6.3 says that $p$ may be satisfied in the $\mathcal{Q}$-power $\mathcal{G}^{n}$ for some $n \geq 1\left(\mathbb{Q}^{n}\right.$, if $\left.G=\{0\}\right)$. Since $\mathcal{G}$ is Archimedean, $\mathcal{G}^{n}\left(\mathbb{Q}^{n}\right)$ is simple; so since $v>0$ in $\mathcal{G}^{n}\left(\mathbb{Q}^{n}\right)$, there is $m \geq 1$ for which

$$
-m v<d<m v
$$

in $\mathcal{G}^{n}\left(\mathbb{Q}^{n}\right)$. So $p \cup\{-m v<d<m v\}$ is a condition and the argument is complete.

So

Theorem 6.5. If $\mathcal{G}$ is Archimedean, then there is a simple dimension group that is $\mathcal{G}$-finitely generic.

Such a dimension group will be a model of the $\forall \exists$-theory $T_{\mathcal{G}}^{\mathcal{Q}}$ that is $\mathcal{G}$-e.c. and so e.c.

Proof. Using the terminology of ([5], Sections 2.2 and 2.3) one may describe a winning strategy for $\exists$, who plays, first to produce a dimension group finitely generic with respect to $T_{\mathcal{G}}^{\mathcal{Q}}$, and then to force

$$
\forall x \bigvee_{m \geq 1}-m v \leq x \leq m v
$$

whenever $v>0$ appears in a condition produced by $\forall$. The compiled structure will be a $\mathcal{G}$-e.c. dimension group in which every positive element is an order unit. Therefore the group has no nontrivial ideals and is simple.

By showing that the property of being simple is elementary within the class of e.c. dimension groups, the next few results will imply that all finitely generic dimension groups are simple.

Lemma 6.6. If $a, b \mid 0$ in the divisible dimension group $\mathcal{D}$, then $b=$ ra for some $r>0$ in $\mathbb{Q}$ just in case

$$
\left(\bigvee_{\bar{m} \in \mathbb{N}^{2} \backslash\{\overline{0}\}} 0 \leq m_{1}(-a)+m_{2} b\right) \wedge\left(\bigvee_{\bar{n} \in \mathbb{N}^{2} \backslash\{\overline{0}\}} 0 \leq n_{1} a+n_{2}(-b)\right)
$$

Proof. If $b=r a$, where $r=m / n$ and $0<m, n \in \mathbb{N}$, then $0=m a-n b$ and so

$$
0 \leq m(-a)+n b \wedge 0 \leq m a+n(-b) .
$$

Conversely, suppose that

$$
0 \leq m_{1}(-a)+m_{2} b \wedge 0 \leq n_{1} a+n_{2}(-b)
$$

for some $\bar{m}, \bar{n} \in \mathbb{N}^{2} \backslash\{\overline{0}\}$. Since

$$
a \mid 0 \text { iff }(-a) \mid 0
$$


and $\mathcal{D}$ has isolated order, neither $m_{2}$ nor $n_{2}$ is 0 ; similarly neither $m_{1}$ nor $n_{1}$ is 0 , and all the $m$ s and $n$ s are positive. Because

$$
m_{1} a \leq m_{2} b \text { and } n_{2} b \leq n_{1} a,
$$

one concludes that for $r=n_{1} / n_{2}$ and $s=m_{2} / m_{1}$,

$$
a \leq s b, b \leq r a \text {, and } a \leq r s a \text {. }
$$

$r s>0$. If $r s>1$, then $0 \leq(r s-1) a$ and $0 \leq a$; if $r s<1$, then $(1-r s) a \leq 0$ and $a \leq 0$. Because $a \mid 0, r s=1, b \leq r a \leq b$, and $b=r a$.

So with the help of schema (3) one concludes that

Corollary 6.7. If $a, b \mid 0$ in the e.c. dimension group $\mathcal{D}$, then $b=$ ra for some $r>0$ in $\mathbb{Q}$ just in case

$$
\forall z(-a, b<z \rightarrow 0<z) \wedge \forall z(a,-b<z \rightarrow 0<z) .
$$

Thus

Corollary 6.8. There is a universal $\mathcal{Q}$-formula $q_{\mid}(x, y)$ such that if a $\mid 0$ in the e.c. dimension group $\mathcal{D}$, then $q_{\mid}(a, y)$ defines $\mathbb{Q} \cdot a$.

Proof. When $a \mid 0$ in $\mathcal{D},(\mathbb{Q} \cdot a) \backslash\{0\}$ consists of elements incomparable with 0 , and so one may apply Corollary 6.7 $q_{\mid}(x, y)$ may be the following formula:

$$
\begin{aligned}
y \neq 0 \rightarrow y \mid 0 \wedge( & {[\forall z(-x, y<z \rightarrow 0<z) \wedge \forall z(x,-y<z \rightarrow 0<z)] } \\
& \vee[\forall z(x, y<z \rightarrow 0<z) \wedge \forall z(-x,-y<z \rightarrow 0<z)]) .
\end{aligned}
$$

The next few results show that $\mathbb{Q} \cdot a$ is definable in e.c. dimension groups when $a>0$. First, note that

Lemma 6.9. In any e.c. dimension group,

$$
\forall z>0 \exists x, y(z=x+y \wedge x, y, x-y \mid 0) .
$$

Proof. Suppose $0<a$ in the e.c. dimension group $\mathcal{D}$. The diagonal map $\delta: D \rightarrow D^{2}$ embeds $\mathcal{D}$ in its $\mathcal{Q}$-power $\mathcal{D}^{2}$, where $\delta(a)=(a, 0)+(0, a)$ and $(a, 0),(0, a)$, and $(a, 0)-(0, a)=(a,-a)$ are incomparable with $(0,0)$. Because $\mathcal{D}$ is e.c. the desired conclusion follows.

Lemma 6.9 will allow one to apply

Lemma 6.10. There is an $\exists \forall$-formula $\varphi_{>}(x, y, z, v, w)$ such that in any e.c. $d i$ mension group $\mathcal{D}$ with elements $a, b, c$ obeying

$$
0<a=b+c \text { and } b, c, b-c \mid 0,
$$

$\varphi_{>}(a, b, c, v, w)$ defines the linear function $f: \mathbb{Q} \cdot b \rightarrow \mathbb{Q} \cdot c$ that sends $b$ to $c$.

Proof. $\varphi_{>}(x, y, z, v, w)$ may be

$$
v \in \mathbb{Q} \cdot y \wedge w \in \mathbb{Q} \cdot z \wedge \exists t \in \mathbb{Q} \cdot(y-z)(v=w+t):
$$

i.e.,

$$
q_{\mid}(y, v) \wedge q_{\mid}(z, w) \wedge \exists t\left(q_{\mid}(y-z, t) \wedge v=w+t\right) .
$$

Suppose $0<a=b+c$ and $b, c, b-c \mid 0$ in the e.c. dimension group $\mathcal{D}$. Since $\mathbb{Q} \cdot b$ and $\mathbb{Q} \cdot c$ are one-dimensional vector spaces over $\mathbb{Q}$, there is a linear function $f: \mathbb{Q} \cdot b \rightarrow \mathbb{Q} \cdot c$ sending $b$ to $c$. For all $r \in \mathbb{Q}$,

$$
r b=r c+r(b-c)
$$


so $\varphi_{>}(a, b, c, g, f(g))$ for all $g \in \mathbb{Q} \cdot b$. Conversely, suppose $\varphi_{>}(a, b, c, g, h)$, and pick $r, s, t \in \mathbb{Q}$ with

$$
g=r b, h=s c, \text { and } r b=s c+t(b-c) .
$$

Then $(r-t) b=(s-t) c$. If $r \neq t$, then $b \in \mathbb{Q} \cdot c, a=b+c \in \mathbb{Q} \cdot c$, and since $a \neq 0, c$ is a rational multiple of $a>0$ and is comparable with 0 . This contradiction implies that $r=t$; so $0=(s-t) c, s=t$, and

$$
h=s c=f(s b)=f(r b)=f(g) .
$$

The last two lemmas yield the following analogue of Corollary 6.8.

Lemma 6.11. There is an $\exists \forall$-formula $q_{>}(x, y)$ such that if $a>0$ in the e.c. dimension group $\mathcal{D}$, then $q_{>}(a, y)$ defines $\mathbb{Q} \cdot a$.

Proof. Note that if $0<a=b+c$, where $b, c, b-c \mid 0$, and $f: \mathbb{Q} \cdot b \rightarrow \mathbb{Q} \cdot c$ is the linear function sending $b$ to $c$, then

$$
\mathbb{Q} \cdot a=\{r b+r c: r \in \mathbb{Q}\}=\{g+f(g): g \in \mathbb{Q} \cdot b\} .
$$

So $q_{>}(x, y)$ may be the formula

$$
\exists y^{\prime}, z^{\prime}\left(x=y^{\prime}+z^{\prime} \wedge y^{\prime}, z^{\prime}, y^{\prime}-z^{\prime} \mid 0 \wedge \exists v, w\left(\varphi>\left(x, y^{\prime}, z^{\prime}, v, w\right) \wedge y=v+w\right)\right) .
$$

So one concludes that

Corollary 6.12. There is an $\forall \exists \forall$-sentence $\sigma$ that is true in an e.c. dimension group just in case it is simple.

Proof. Informally, $\sigma$ is

$$
\forall x>0 \forall y \exists z \in \mathbb{Q} \cdot x(-z \leq y \leq z):
$$

i.e.,

$$
\forall x>0 \forall y \exists z\left(q_{>}(x, z) \wedge-z \leq y \leq z\right) .
$$

As noted before, the completeness of the theory of finitely generic dimension groups combines with Theorem 6.5 to yield

Theorem 6.13. All finitely generic dimension groups are simple.

One may conclude also that

Theorem 6.14. No infinitely generic dimension group is simple.

Proof. Let $\mathcal{G}$ be a dimension group that is not simple: a non-Archimedean ordered Abelian group, for example. There is an infinitely generic $\mathcal{H} \supseteq \mathcal{G}$ ([5], pp. 153-157), and by Corollary 6.12, $\sigma$ fails in $\mathcal{H}$. Because all infinitely generic dimension groups are elementarily equivalent, $\sigma$ fails in all infinitely generic dimension groups.

Despite the last two theorems, simple e.c. dimension groups need not be finitely generic. Much as before one may approach this result by building a special finitely generic dimension group.

Lemma 6.15. In finite forcing relative to $T^{\mathcal{Q}}$,

$$
\{v \neq 0\} \Vdash \forall x \bigvee_{q \in \mathbb{Q}}(x \mid q v \vee x=q v)
$$


Proof. As in the proof of Lemma 6.4 one shows that if $p \supseteq\{v \neq 0\}$ is a condition and $d \in W$ is not in $p$, then there are a rational $q$ and a condition $r \supseteq p$ with $r \Vdash d \mid q v \vee d=q v$. Lemma 6.3 allows one to satisfy $p$ in some $\mathcal{Q}$-power $\mathbb{Q}^{n}$ of $\mathbb{Q}$. $v \neq 0$ in $\mathbb{Q}^{n}$; so some coordinate $v_{i}$ of $v$ is nonzero, and there is $q \in \mathbb{Q}$ such that the $i$ th coordinate $d_{i}$ of $d$ is $q v_{i}$. If $d \neq q v$ in $\mathbb{Q}^{n}$, then $d \mid q v$ in $\mathbb{Q}^{n}$; so at least one of $p \cup\{d=q v\}, p \cup\{d \mid q v\}$ is a condition and the argument is complete.

Corollary 6.16. In every finitely generic dimension group,

$$
\forall z>0 \forall x(\forall w \in \mathbb{Q} \cdot z(x \nmid w) \rightarrow x \in \mathbb{Q} \cdot z) .
$$

Proof. Because all finitely generic dimension groups are elementarily equivalent and Lemma 6.11 is available, one need show merely that some finitely generic dimension group has the given property. Reverting to the terminology of [5], let $\exists$ play to produce a finitely generic dimension group and to force

$$
\forall x \bigvee_{q \in \mathbb{Q}}(x \mid q v \vee x=q v)
$$

whenever $v>0$ appears in a condition produced by $\forall$. The compiled structure is a finitely generic dimension group in which, if $a>0$ and $b$ is comparable to every element of $\mathbb{Q} \cdot a$, then $b \in \mathbb{Q} \cdot a$ because

$$
\bigvee_{q \in \mathbb{Q}}(b \mid q a \vee b=q a) .
$$

An easy argument now implies that

Corollary 6.17. There are simple e.c. dimension groups that are not finitely generic.

Proof. Starting with the Archimedean ordered Abelian group $\mathcal{G}=\mathbb{Q}(\sqrt{2})$, invoke Theorem 6.5 to obtain a simple $\mathcal{G}$-finitely generic dimension group $\mathcal{H}$. Since $\mathcal{G} \subseteq \mathcal{H}$, $\mathcal{H}$ is e.c. In $\mathcal{H}, \sqrt{2}$ is comparable with every element of $\mathbb{Q} \cdot 1$ but does not belong to $\mathbb{Q} \cdot 1$; so by Corollary 6.16, $\mathcal{H}$ is not finitely generic.

Because the most obvious way to create many different quantifier-free types is to fill cuts in the rationals, Corollary 6.16 leave mysterious the structure of the quantifier-free 2-type of $(a, b)$ when $a>0$. Some of the mystery is dispelled by

Theorem 6.18. In a finitely generic dimension group,

$$
\begin{aligned}
\forall x>0 \forall y \forall z \in \mathbb{Q} \cdot x[ & (y<z \rightarrow \exists w \in \mathbb{Q} \cdot x(y<w<z)) \\
& \wedge(z<y \rightarrow \exists w \in \mathbb{Q} \cdot x(z<w<y)) \\
& \wedge(y \mid z \rightarrow \exists v, w \in \mathbb{Q} \cdot x(v<z<w \wedge y \mid v, w))] .
\end{aligned}
$$

Given this result, one may describe as follows the quantifier-free type of $(a, b)$ when $a>0$ in the finitely generic dimension group $\mathcal{M}$. One possibility is that $b=r a$ for some $r \in \mathbb{Q}$. If this case does not happen, then since $\mathcal{M}$ is simple and $a>0$ there are $r<s$ in $\mathbb{Q}$ with $r a<b<s a$, and by Corollary 6.16 there is $t \in \mathbb{Q}$ with $b \mid t a$. So $\mathbb{Q}$ admits the partition

$$
\mathbb{Q}=\{r \in \mathbb{Q}: r a<b\} \amalg\{t \in \mathbb{Q}: b \mid t a\} \amalg\{s \in \mathbb{Q}: b<s a\},
$$

and since $a>0$ all elements of the first set are less than all elements of the second set which are less than all elements of the third set. Theorem 6.18 implies that all 
these sets are open in $\mathbb{Q}$; so there are irrational $\alpha<\beta$ in $\mathbb{R}$ such that for all rational $r, s$, and $t$,

$$
r a<b \text { iff } r<\alpha, b \mid t a \text { iff } \alpha<t<\beta, \text { and } b<s a \text { iff } \beta<s .
$$

This phenomenon does not occur in $\mathbb{Q}^{2}$, for example, where $(1,1)>(0,0)$ and

$$
r(1,1)<(0,1) \text { iff } r<0,(0,1) \mid t(1,1) \text { iff } 0 \leq t \leq 1 \text {, and }(0,1)<s(1,1) \text { iff } 1<s .
$$

So $\mathbb{Q}^{2}$ cannot be embedded in a finitely generic dimension group $\mathcal{M}$.

On the basis of Lemma 6.11 one may prove Theorem 6.18 by showing that for every $r \in \mathbb{Q}$, the empty condition forces each of

(1) $\forall x>0 \forall y\left(y<r x \rightarrow \bigvee_{s<r} y<s x\right)$,

(2) $\forall x>0 \forall y\left(r x<y \rightarrow \bigvee_{r<s} s x<y\right)$, and

(3) $\forall x>0 \forall y\left(y\left|r x \rightarrow \bigvee_{s<r<t} y\right| s x, t x\right)$.

Let $a$ and $b$ be distinct witnesses.

The empty condition will force (1) if every condition $p$ extending $\{a>0, b<r a\}$ is consistent with $b<s a$ for some $s<r$. $p$ may be satisfied in $\mathbb{Q}^{k}$ for some positive integer $k$. For $i=1, \ldots, k$,

$$
a_{i}>0 \text { and } b_{i}<r a_{i}
$$

so there is $s<r$ with $b_{i}<s a_{i}$ for $i=1, \ldots, k$. Thus $p \cup\{b<s a\}$ is satisfied in $\mathbb{Q}^{k}$, as desired.

A similar argument shows that the empty condition forces (2).

Suppose now that $p$ is a condition extending $\{a>0, b \mid r a\}$, and as before assume that $p$ is satisfied in $\mathbb{Q}^{k}$. If there are $i, j \in\{1, \ldots, k\}$ with

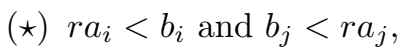

then since every $a_{l}>0$ there are $s<r<t$ in $\mathbb{Q}$ with

$$
s a_{i}<r a_{i}<t a_{i}<b_{i} \text { and } b_{j}<s a_{j}<r a_{j}<t a_{j},
$$

and $p \cup\{b \mid s a, t a\}$ is satisfied in $\mathbb{Q}^{k}$. If there are no $i, j \in\{1, \ldots, k\}$ obeying $(\star)$, then one may without loss of generality suppose that $r a_{i} \leq b_{i}$ for all $i, r a_{j}=b_{j}$ for some $j$, and $r a_{l}<b_{l}$ for some $l$. If $p$ contains an identity with nontrivial occurrences of witnesses other than $a$ and $b$, one may solve for such a witness - $c$, say-in terms of the others in the identity and then eliminate $c$ from $p$. Repeating this process as often as possible, one obtains a condition $q$, satisfied in $\mathbb{Q}^{k}$, that contains no identities with nontrivial occurrences of witnesses other than $a$ and $b$. If one can find $s<r<t$ in $\mathbb{Q}$ for which $q \cup\{b \mid s a, t a\}$ is a condition, $p \cup\{b \mid s a, t a\}$ also will be a condition, since the previously eliminated witnesses may be restored with the help of the identities used to eliminate these witnesses. Because $\{a>0, b \mid r a\} \subseteq q$, it contains no nontrivial identities, and without loss of generality consists of (strict) inequalities, negations of inequalities, and negations of identities; let $q^{\prime}$ consist of all the inequalities in $q$. Since $r a_{j}=b_{j}, q^{\prime} \cup\{r a=b\}$ is consistent with the $\mathcal{Q}$-theory of divisible ordered Abelian groups; so since $q^{\prime}$ consists of (strict) inequalities, there are $s<r<t$ in $\mathbb{Q}$ such that both $q^{\prime} \cup\{s a=b\}$ and $q^{\prime} \cup\{t a=b\}$ are consistent with the theory of divisible ordered Abelian groups. Thus $q^{\prime} \cup\{s a=b\}$ and $q^{\prime} \cup\{t a=b\}$ may be satisfied in $\mathbb{Q}$, and one may adjoin corresponding assignments in $\mathbb{Q}$ to the earlier assignment in $\mathbb{Q}^{k}$ to obtain an assignment in $\mathbb{Q}^{k+2}$. This assignment satisfies $q \cup\{b \mid s a, t a\}$ : the assignments in $\mathbb{Q}^{k}$ and $\mathbb{Q}$ satisfy $q^{\prime} ;$ the assignment in $\mathbb{Q}^{k}$ satisfies the negated-atomic formulas of $q \backslash q^{\prime}$; since $s<r$ and $a_{j}>0, b_{j}=r a_{j}>s a_{j}$, and 
so $b \mid s a$ since $b_{k+1}=s a_{k+1}$; since $a_{k+1}>0$ and $s<t, t a_{k+1}>s a_{k+1}=b_{k+1}$ and so $b \mid t a$ since $b_{k+2}=t a_{k+2}$.

A different forcing argument reveals another way in which finitely generic dimension groups are small. Assume, for the moment,

Lemma 6.19. If $v, v^{\prime}$ are witnesses, $\left\{v, v^{\prime}>0\right\}$ forces

$$
\begin{gathered}
\forall y, y^{\prime}\left[\bigvee_{\bar{r} \in \mathbb{Q}^{3}} y=r_{1} v+r_{2} v^{\prime}+r_{3} y^{\prime} \vee \bigvee_{\bar{r} \in \mathbb{Q}^{3} \backslash\{\overline{0}\}} r_{1} v+r_{2} y+r_{3} y^{\prime}=0\right. \\
\left.\vee \bigvee_{r \in \mathbb{Q}} \neg\left(r v<y \leftrightarrow r v^{\prime}<y^{\prime}\right)\right] .
\end{gathered}
$$

Then one may show that

Corollary 6.20. There is a finitely generic dimension group isomorphic to no proper substructure of itself.

Proof. Let $\exists$ play to produce a finitely generic dimension group and to force the formula just displayed whenever $v, v^{\prime}>0$ appear in a condition produced by $\forall$. The compiled structure $\mathcal{D}$ will be finitely generic and have the following property: if $a, a^{\prime}>0$ and $b, b^{\prime}$ all come from $D, b$ is not in the linear span of $a, a^{\prime}, b^{\prime}$, and $a, b, b^{\prime}$ are linearly independent, then $(a, b)$ and $\left(a^{\prime}, b^{\prime}\right)$ have different quantifier-free types in $\mathcal{D}$.

Suppose now that $\varphi: \mathcal{D} \cong \mathcal{E} \subset \mathcal{D}$. Pick $a>0$ in $E$. Because the elements incomparable with 0 generate $\mathcal{D}$ as a group (Lemma 6.9), there is $b \in D \backslash E$ with $b \mid 0 ;$ let $\left(a^{\prime}, b^{\prime}\right)=(\varphi(a), \varphi(b)) . a^{\prime}=\varphi(a)>0$, and since $b \in D \backslash E$ and $a, a^{\prime}, b^{\prime} \in E$, a proper subspace of $D, b$ is not in the linear span of $a, a^{\prime}, b^{\prime}$. Since $a>0$ and $b^{\prime}=\varphi(b) \mid 0, a$ and $b^{\prime}$ are linearly independent, and so $a, b, b^{\prime}$ are linearly independent since $b$ is not in the linear span of $a$ and $b^{\prime}$. Thus $(a, b)$ and $\left(a^{\prime}, b^{\prime}\right)$ have different quantifier-free types in $\mathcal{D}$, and since $\left(a^{\prime}, b^{\prime}\right) \in E^{2}$ has the same quantifier-free type in $\mathcal{D}$ and in $\mathcal{E} \subset \mathcal{D}, \varphi$ changes the quantifier-free type of $(a, b)$. This contradiction gives the result desired.

Before showing that all finitely generic dimension groups obey the conclusion of Corollary 6.20, one should prove Lemma 6.19. One need show merely that if $w, w^{\prime}$ are distinct witnesses different from $v, v^{\prime}$ and the condition $p$ extends $\left\{v, v^{\prime}>0\right\}$, then $p$ is consistent with $w=r_{1} v+r_{2} v^{\prime}+r_{3} w^{\prime}$ for some $\bar{r} \in \mathbb{Q}^{3}$, with $r_{1} v+r_{2} w+$ $r_{3} w^{\prime}=0$ for some $\bar{r} \in \mathbb{Q}^{3} \backslash\{\overline{0}\}$, or with $\neg\left(r v<w \leftrightarrow r v^{\prime}<w^{\prime}\right)$ for some $r \in \mathbb{Q} . p$ may be satisfied in some $\mathbb{Q}^{k}$. If under this interpretation $w$ is a linear combination of $v, v^{\prime}, w^{\prime}$ or $v, w, w^{\prime}$ are linearly dependent, the desired conclusion follows; so assume that neither of these relationships holds. If $v, v^{\prime}, w, w^{\prime}$ are linearly independent in $\mathbb{Q}^{k}$, then as in the proof of Theorem 6.18 one may eliminate identities from $p$ to obtain a condition $q \supseteq\left\{v, v^{\prime}>0\right\}$, satisfied in $\mathbb{Q}^{k}$, such that $p \cup\{\neg(r v<w \leftrightarrow$ $\left.\left.r v^{\prime}<w^{\prime}\right)\right\}$ is consistent whenever $q \cup\left\{\neg\left(r v<w \leftrightarrow r v^{\prime}<w^{\prime}\right)\right\}$ is consistent. If $v, v^{\prime}, w, w^{\prime}$ are linearly dependent in $\mathbb{Q}^{k}$, then since $w$ is not in the span of $v, v^{\prime}, w^{\prime}$ in $\mathbb{Q}^{k}, v, v^{\prime}, w^{\prime}$ are linearly dependent in $\mathbb{Q}^{k}$; so since $v, w^{\prime}$ are linearly independent in $\mathbb{Q}^{k}$ there is a linear form $f$ in $v, w^{\prime}$ such that $v^{\prime}=f$ in $\mathbb{Q}^{k}$. Use this identity to eliminate $v^{\prime}$ from $p$. If it contains any other nontrivial identities, they must involve some witness other than $v, w, w^{\prime}$, and one may as before eliminate such a witness, and the corresponding identity, from $p$. One thus obtains a condition $q$, extending $\{v>0, f>0\}$ and satisfied in $\mathbb{Q}^{k}$, that contains no identities and has the property 
that $p \cup\left\{\neg\left(r v<w \leftrightarrow r v^{\prime}<w^{\prime}\right)\right\}$ is consistent whenever $q \cup\left\{\neg\left(r v<w \leftrightarrow r f<w^{\prime}\right)\right\}$ is consistent. So whether or not $v, v^{\prime}, w, w^{\prime}$ are linearly independent in $\mathbb{Q}^{k}$, there are a linear form $f$ in $v, v^{\prime}, w^{\prime}$ and a condition $q \supseteq\{v>0, f>0\}$, satisfied in $\mathbb{Q}^{k}$, that contains no identities and has the property that $p \cup\left\{\neg\left(r v<w \leftrightarrow r v^{\prime}<w^{\prime}\right)\right\}$ is consistent whenever $q \cup\left\{\neg\left(r v<w \leftrightarrow r f<w^{\prime}\right)\right\}$ is consistent. Without loss of generality,

$$
r v<w \text { in } \mathbb{Q}^{k} \text { iff } r f<w^{\prime} \text { in } \mathbb{Q}^{k}
$$

for all $r \in \mathbb{Q}$. Because $v, f>0$ in $\mathbb{Q}^{k}$, there is $r_{0} \in \mathbb{Q}$ with

$$
r_{0}=\operatorname{lub}\left\{r \in \mathbb{Q}: r v<w \text { in } \mathbb{Q}^{k}\right\}=\operatorname{lub}\left\{r \in \mathbb{Q}: r f<w^{\prime} \text { in } \mathbb{Q}^{k}\right\} .
$$

In some coordinate - say the $i$ th $-r_{0} v_{i}=w_{i}$. If $q^{\prime}$ consists of all the inequalities in $q$, then the restriction $\rho$ of the assignment in $\mathbb{Q}^{k}$ to the $i$ th coordinate satisfies $q^{\prime} \cup\left\{r_{0} v=w\right\}$. Because $q^{\prime}$ consists of inequalities, one may change, in $\rho$, just the value of $w$ to obtain a new $\mathbb{Q}$-assignment $\sigma$ satisfying $q^{\prime} \cup\{r v=w\}$ for some $r<r_{0}$. Since $\rho$ makes $r f<w^{\prime}$, the assignments $\rho$ and $\sigma$ agree on all witnesses except $w$, and $f$ is a linear form in $v, v^{\prime}$, and $w^{\prime}, \sigma$ makes $r f<w^{\prime}$. So if one expands the assignment in $\mathbb{Q}^{k}$ to an assignment in $\mathbb{Q}^{k+1}$ by using $\sigma$ in the last coordinate, one obtains an assignment satisfying $q \cup\left\{\neg\left(r v<w \leftrightarrow r f<w^{\prime}\right)\right\}$, and the argument is complete.

To show that all finitely generic dimension groups obey the conclusion of Corollary 6.20 one must define certain notions in e.c. dimension groups. First one may state

Lemma 6.21. There is an $\exists \forall$-formula $q(x, y)$ such that for any element a of an e.c. dimension group $\mathcal{D}, q(a, y)$ defines $\mathbb{Q} \cdot a$.

Proof. If $q_{\mid}(x, y)$ and $q_{>}(x, y)$ are the formulas from Corollary 6.8 and Lemma 6.11. $q(x, y)$ may be

$$
x=y=0 \vee\left(x>0 \wedge q_{>}(x, y)\right) \vee\left(x<0 \wedge q_{>}(-x, y)\right) \vee\left(x \mid 0 \wedge q_{\mid}(x, y)\right) .
$$

Next one may state a variant of Lemma 6.10,

Lemma 6.22. There is an $\exists \forall$-formula $\varphi\left(x, x^{\prime}, v, w\right)$ such that if $a, a^{\prime}$ are linearly independent elements of an e.c. dimension group $\mathcal{D}$, then $\varphi\left(a, a^{\prime}, v, w\right)$ defines in $\mathcal{D}$ the linear function $f: \mathbb{Q} \cdot a \rightarrow \mathbb{Q} \cdot a^{\prime}$ that sends a to $a^{\prime}$.

Proof. $\varphi\left(x, x^{\prime}, v, w\right)$ may be

$$
v \in \mathbb{Q} \cdot x \wedge w \in \mathbb{Q} \cdot x^{\prime} \wedge \exists z \in \mathbb{Q} \cdot\left(x-x^{\prime}\right)(v=w+z):
$$

i.e.,

$$
q(x, v) \wedge q\left(x^{\prime}, w\right) \wedge \exists z\left(q\left(x-x^{\prime}, z\right) \wedge v=w+z\right)
$$

The argument that this formula works resembles that for Lemma 6.10.

A generalization of Lemma 6.22 rests on

Lemma 6.23. No e.c. dimension group is finite-dimensional.

Proof. If $\mathcal{D}$ is an e.c. dimension group and $n$ is a positive integer, the diagonal map $\delta: \mathcal{D} \rightarrow \mathcal{D}^{n+1}$ embeds $\mathcal{D}$ in its $\mathcal{Q}$-power $\mathcal{D}^{n+1}$. Pick $d>0$ in $D$, and for $i=1, \ldots, n$ let $d_{i} \in \mathcal{D}^{n+1}$ be zero in every coordinate but the $i$ th, where the value is $d$. Since

$$
\bigwedge_{\bar{m} \in \mathbb{Z}^{n} \backslash\{\overline{0}\}} 0 \not \leq \sum_{i} m_{i} d_{i},
$$


one may argue as in Lemma 5.3 that in any e.c. extension of $\mathcal{D}^{n+1}$,

$$
\exists x_{1} \ldots \exists x_{n} \bigwedge_{j:\{1, \ldots, n\} \rightarrow\{0,1\}} \exists y\left(\bigwedge_{i=1}^{n}(-1)^{j(i)} x_{i}<y \wedge 0 \nless y\right) .
$$

So the e.c. structure $\mathcal{D}$ obeys this sentence, and if $e_{1}, \ldots, e_{n} \in D$ obey

$$
\bigwedge_{j:\{1, \ldots, n\} \rightarrow\{0,1\}} \exists y\left(\bigwedge_{i=1}^{n}(-1)^{j(i)} e_{i}<y \wedge 0 \nless y\right)
$$

in $\mathcal{D}$, then

$$
\bigwedge_{\bar{m} \in \mathbb{Z}^{n} \backslash\{\overline{0}\}} 0 \not \leq \sum_{i} m_{i} e_{i}
$$

and $e_{1}, \ldots, e_{n}$ are linearly independent. $n$ being arbitrary, the desired result follows.

The last two lemmas imply that

Lemma 6.24. There is an $\exists \forall \exists$-formula $\gamma\left(x, x^{\prime}, v, w\right)$ such that for all nonzero elements $a, a^{\prime}$ of an e.c. dimension group $\mathcal{D}, \gamma\left(a, a^{\prime}, v, w\right)$ defines the linear function $f: \mathbb{Q} \cdot a \rightarrow \mathbb{Q} \cdot a^{\prime}$ that sends a to $a^{\prime}$.

Proof. $\gamma\left(x, x^{\prime}, v, w\right)$ may be

$$
\exists x^{\prime \prime}\left(x^{\prime \prime} \notin \mathbb{Q} \cdot x \wedge x^{\prime \prime} \notin \mathbb{Q} \cdot x^{\prime} \wedge \exists z\left(\varphi\left(x, x^{\prime \prime}, v, z\right) \wedge \varphi\left(x^{\prime \prime}, x^{\prime}, z, w\right)\right)\right) .
$$

Putting all these results together, one concludes that

Theorem 6.25. There is an $\forall \exists \forall \exists$-sentence ८ which is true in an e.c. dimension group $\mathcal{D}$ just in case it has the following property: if $a, a^{\prime}>0$ and $b, b^{\prime}$ are from $D$, $b$ is not in the linear span of $a, a^{\prime}, b^{\prime}$, and $a, b, b^{\prime}$ are linearly independent, then for some $r \in \mathbb{Q}$,

$$
\neg\left(r a<b \leftrightarrow r a^{\prime}<b^{\prime}\right) .
$$

Proof. $\iota$ may be the sentence

$$
\begin{aligned}
\forall x, x^{\prime}>0 \forall y, y^{\prime} & {\left[y \in \mathbb{Q} \cdot x+\mathbb{Q} \cdot x^{\prime}+\mathbb{Q} \cdot y^{\prime} \vee x \in \mathbb{Q} \cdot y+\mathbb{Q} \cdot y^{\prime}\right.} \\
& \vee y \in \mathbb{Q} \cdot x+\mathbb{Q} \cdot y^{\prime} \vee y^{\prime} \in \mathbb{Q} \cdot x+\mathbb{Q} \cdot y \\
& \left.\vee \exists v, w\left(\gamma\left(x, x^{\prime}, v, w\right) \wedge \neg\left(v<y \leftrightarrow w<y^{\prime}\right)\right)\right] .
\end{aligned}
$$

Because all finitely generic dimension groups are elementarily equivalent, one may combine Theorem 6.25 with the proof of Corollary 6.20 to conclude that

Corollary 6.26. Every finitely generic dimension group obeys $\iota$ and so is isomorphic to no proper $\mathcal{Q}$-substructure of itself.

The last two results combine to show that

Theorem 6.27. Every finitely generic dimension group has cardinality at most $2^{\aleph_{0}}$.

Proof. Suppose otherwise, and let $\mathcal{M}$ be a finitely generic dimension group of cardinality at least $\left(2^{\aleph_{0}}\right)^{+}$. Build a chain $\left\{\mathcal{M}_{\alpha}\right\}_{\alpha<\left(2^{\aleph_{0}}\right)^{+}}$of elementary submodels of $\mathcal{M}$, with every $\left|\mathcal{M}_{\alpha}\right|<\left(2^{\aleph_{0}}\right)^{+}$, as follows. $\mathcal{M}_{0} \preccurlyeq \mathcal{M}$ may be any elementary submodel of cardinality less than $\left(2^{\aleph_{0}}\right)^{+}$. If $\lambda<\left(2^{\aleph_{0}}\right)^{+}$is a limit ordinal and the 
chain $\left\{\mathcal{M}_{\alpha}\right\}_{\alpha<\lambda}$ with each $\mathcal{M}_{\alpha} \preccurlyeq \mathcal{M}$ of cardinality $<\left(2^{\aleph_{0}}\right)^{+}$is available, one may let

$$
\mathcal{M}_{\lambda}=\bigcup_{\alpha<\lambda} \mathcal{M}_{\alpha}
$$

which will have cardinality $<\left(2^{\aleph_{0}}\right)^{+}$because this cardinal is regular. Finally, if $\alpha<\left(2^{\aleph_{0}}\right)^{+}$and $\mathcal{M}_{\alpha} \preccurlyeq \mathcal{M}$ has cardinality $<\left(2^{\aleph_{0}}\right)^{+}$, then since $\mathcal{M}$ is generated by the elements incomparable with 0 (Lemma[6.9) there are $b_{\alpha} \mid 0$ with $b_{\alpha} \in M \backslash M_{\alpha}$ and $\mathcal{M}_{\alpha+1} \preccurlyeq \mathcal{M}$ with $M_{\alpha} \cup\left\{b_{\alpha}\right\} \subseteq M_{\alpha+1}$ and $\left|M_{\alpha+1}\right|=\left|M_{\alpha}\right|$. Because each $\mathcal{M}_{\alpha} \preccurlyeq \mathcal{M}$, each $\mathcal{M}_{\alpha}$ is finitely generic ([5], Exercise 4.3.4(c)); so Corollary 6.26 gives each $\mathcal{M}_{\alpha}$ the property described in Theorem 6.25. Fixing $a>0$ in $M_{0}$, one may argue as in Corollary 6.20 to show that the quantifier-free type of $\left(a, b_{\alpha}\right)$ in $\mathcal{M}_{\alpha+1}$ is not realized in $\mathcal{M}_{\alpha}$. So the quantifier-free type of $\left(a, b_{\alpha}\right)$ in $\mathcal{M}_{\alpha+1}$ is not realized in $\mathcal{M}_{\beta}$ if $\beta \leq \alpha$, and one obtains $\left(2^{\aleph_{0}}\right)^{+}$different quantifier-free 2-types for $T^{\mathcal{Q}}$, a theory in a countable language. This contradiction yields the result desired.

By exploiting results from [7] and 2] on the quantifier "there are uncountably many $x "(\mathrm{Q} x)$ one may show that

Theorem 6.28. Every countable finitely generic dimension group may be embedded in $2^{\aleph_{1}}$ pairwise nonembeddable finitely generic dimension groups of cardinality $\aleph_{1}$.

Proof. Let $\mathcal{M}$ be a countable finitely generic dimension group. Note first that by ([5], Exercises 4.3.3-4.3.4) the class of finitely generic dimension groups has a countable set $G$ of axioms in $\mathcal{Q}_{\omega_{1} \omega}$, each of the form

$$
\forall \bar{x} \bigvee_{n \in \mathbb{N}} \psi_{n}(\bar{x})
$$

where the $\psi$ s are existential quantifications of finite conjunctions of atomic and negated-atomic formulas. Let $G_{\mathcal{M}}=G \cup \operatorname{diag}(\mathcal{M})$. Suppose one can find $\mathcal{N} \models G_{\mathcal{M}}$ of cardinality $\aleph_{1} . \mathcal{N} \backslash \mathcal{Q} \supseteq \mathcal{M}$ is a finitely generic dimension group of cardinality $\aleph_{1}$, and the argument behind Theorem 6.27 shows that $\mathcal{N}$ realizes $\aleph_{1}$ different quantifier-free 2 -types of $\mathcal{Q}$. So Lemma 5.5 of 7 ] provides a family of $2^{\aleph_{1}}$ models of $G_{\mathcal{M}}$, of cardinality $\aleph_{1}$, such that if $\mathcal{A}$ and $\mathcal{B}$ are different members of the family then $\mathcal{A}$ realizes a quantifier-free 2 -type not realized in $\mathcal{B}$, and the desired result follows.

To find a single model of $G_{\mathcal{M}}$ of cardinality $\aleph_{1}$ one may invoke the "Q-Omitting Types Theorem" of [2]. With the help of ([2], Theorem 1.1 and the Definition on p. 237) one sees that the "Q-Omitting Types Theorem" provides such a model if the new quantifier $\mathrm{Q}$ may be interpreted so that $\mathcal{M}$ satisfies seven schemas of $\mathcal{Q}(\mathrm{Q})_{\omega_{1} \omega}$. These schemas are universal closures of the following formulas, which are subject to the restrictions given below:

(i) $\mathrm{Q} v(v=v)$

(ii) $\neg \mathrm{Q} v(v=w)(v, w$ are different variables $)$

(iii) $\forall v(\varphi \rightarrow \psi) \rightarrow(\mathrm{Q} v \varphi \rightarrow \mathrm{Q} v \psi)$

(iv) $\mathrm{Q} v \gamma(v) \rightarrow \mathrm{Q} w \gamma(w)$ whenever $w$ is free for $v$ in $\gamma(v)$

(v) $\mathrm{Q} v(\varphi \vee \psi) \rightarrow \mathrm{Q} v \varphi \vee \mathrm{Q} v \psi$

(vi) $\mathrm{Q} v \exists w \beta \rightarrow \exists w \mathrm{Q} v \beta \vee \mathrm{Q} w \exists v \beta$

(vii) $\mathrm{Q} v \bigvee_{n \in \mathbb{N}} \beta_{n} \rightarrow \bigvee_{n \in \mathbb{N}} \mathrm{Q} v \beta_{n}$ 
Formulas (i)-(vii) belong to the smallest countable fragment of $\left(\mathcal{Q}(\mathrm{Q})_{M}\right)_{\omega_{1} \omega}([2$, pp. 228-229) that contains $G_{\mathcal{M}}$ and also belong to $\mathcal{Q}(\mathrm{Q})_{\omega_{1} \omega}$; so each formula contains only finitely many free variables and no constants from $\mathcal{Q}_{M} \backslash \mathcal{Q}$. Also, each $\beta$ or $\beta_{n}$ results from a finite conjunction of atomic or negated-atomic $\mathcal{Q}$-formulas when the quantifiers $\mathrm{Q}$ and $\exists$ are applied to certain variables.

Let

$$
\mathcal{M} \models \mathrm{Q} x \varphi(\bar{a}, x) \text { iff }\{b \in M: \mathcal{M} \models \varphi(\bar{a}, b)\} \text { contains an infinite }
$$

linearly independent set.

$\mathcal{M}$ satisfies schema (i) by Lemma 6.23 and schemas (ii)-(v) by definition; extra work is needed to show that (vi) and (vii) are satisfied.

Schemas (iii) and (v) imply that Q, like $\exists$, commutes with finite $\vee$. Because Lemma 3.1 makes any quantifier-free $\mathcal{Q}$-formula equivalent in $\mathcal{M}$ to a finite disjunction of finite conjunctions of identities, inequalities, and incomparabilities, one may assume that in (vi) $\beta$ is $\bigvee_{i=1}^{n} \beta_{i}^{\prime}$, where each $\beta_{i}^{\prime}$ results from a finite conjunction of identities, inequalities, and incomparabilities when quantifiers $\mathrm{Q}$ and $\exists$ are applied to certain variables. Again because $Q$ and $\exists$ commute with finite $\vee, \beta$ will obey (vi) if each $\beta_{i}^{\prime}$ does; so without loss of generality $\beta$ has the form

$$
\mathrm{Q}_{1} y_{1} \ldots \mathrm{Q}_{l} y_{l} \delta(v, w, \bar{x}, \bar{y})
$$

where each $\mathrm{Q}_{i} \in\{\mathrm{Q}, \exists\}$ and $\delta$ is a conjunction of identities, inequalities, and incomparabilities in $v, w, \bar{x}=\left(x_{1}, \ldots, x_{k}\right)$, and $\bar{y}=\left(y_{1}, \ldots, y_{l}\right)$.

Assume temporarily that

Lemma 6.29. Let $\gamma(\bar{x}, \bar{y})$ be a finite conjunction of identities, inequalities, incomparabilities, and formulas $\exists z(\theta \wedge z \mid 0)$, where $\theta$ is a finite conjunction of inequalities in $\bar{x}=\left(x_{1}, \ldots, x_{k}\right), \bar{y}=\left(y_{1}, \ldots, y_{l}\right)$, and the new variable $z$. Let

$$
\zeta(\bar{x})=Q_{1} y_{1} \ldots Q_{l} y_{l} \gamma(\bar{x}, \bar{y})
$$

where each $Q_{i} \in\{Q, \exists\}$. Then

(i) If $\gamma(\bar{x}, \bar{y})$ contains no identities, then $\zeta(\bar{x})$ is equivalent in $\mathcal{M}$ to $\exists \bar{y} \gamma(\bar{x}, \bar{y})$.

(ii) $\zeta(\bar{x})$ is equivalent in $\mathcal{M}$ to $0 \neq 0$ or to $\exists \bar{y} \gamma(\bar{x}, \bar{y})$.

To verify (vi) in $\mathcal{M}$, assume that

$$
\mathcal{M} \models \mathrm{Q} v \exists w \beta(v, w, \bar{a}),
$$

where $\bar{a}=\left(a_{1}, \ldots, a_{k}\right)$ comes from $M . \beta(v, w, \bar{x})$ cannot be equivalent in $\mathcal{M}$ to $0 \neq 0$; so by Lemma 6.29 $\beta(v, w, \bar{x})$ is equivalent in $\mathcal{M}$ to $\exists \bar{y} \delta(v, w, \bar{x}, \bar{y})$. Lemma 3.3 makes $\exists \bar{y} \delta(v, w, \bar{x}, \bar{y})$ equivalent in $\mathcal{M}$ to a conjunction $\xi(v, w, \bar{x})$ of identities, inequalities, incomparabilities, and formulas $\exists z(\theta \wedge z \mid 0)$ as above. If no identities occur in $\xi$, Lemma 6.29 makes $\mathrm{Q} v \exists w \xi, \exists w \mathrm{Q} v \xi$, and $\mathrm{Q} w \exists v \xi$ all equivalent in $\mathcal{M}$ to $\exists v \exists w \xi$, and the desired conclusion follows. Suppose now that some conjuncts of $\xi$ are identities. If none has nontrivial occurrences of $v$ or $w$, the usual prenexing rules 15 applied to $\mathrm{Q} v \exists w \xi$, allow one to assume that no conjunct of $\xi$ is an identity, and then the desired conclusion follows as before. If at least one identity contains both $v$ and $w$, then $\exists w \mathrm{Q} v \xi(v, w, \bar{a})$ fails in $\mathcal{M}$ but

$$
\mathcal{M}=\mathrm{Q} v \exists w \xi(v, w, \bar{a}) \leftrightarrow \mathrm{Q} w \exists v \xi(v, w, \bar{a}),
$$

\footnotetext{
${ }^{15}$ The interpretation of $\mathrm{Q}$ in $\mathcal{M}$ makes $\mathrm{Q} z(\varphi \wedge \psi)$ equivalent to $\varphi \wedge \mathrm{Q} z \psi$ when $z$ has no free occurrences in $\varphi$.
} 
since in $\xi, w$ (resp. $v$ ) is a linear form in $v$ (resp. $w$ ) and the $x$ s. If at least one identity contains $v$ but not $w$, then

$$
\mathcal{M} \not \models \mathrm{Q} v \exists w \xi(v, w, \bar{a}),
$$

contrary to hypothesis. Finally, if at least one identity contains $w$ but not $v$, then there is a term $t(\bar{x})$ such that $\exists w \xi$ is equivalent in $\mathcal{M}$ to $\xi(v, t(\bar{x}), \bar{x})$; so in $\mathcal{M}$, $\mathrm{Q} v \exists w \xi(v, w, \bar{a})$ implies $\mathrm{Q} v \xi(v, t(\bar{a}), \bar{a})$, which in turn implies $\exists w \mathrm{Q} v \xi(v, w, \bar{a})$. Thus schema (vi) follows from Lemma 6.29

One may prove Lemma 6.29 by induction on $l \geq 1$, and the basis of the induction relies on

Lemma 6.30. Let $\gamma(\bar{x}, y)$ be the $\mathcal{Q}$-formula

$$
\bigwedge_{i} a_{i}<y \wedge \bigwedge_{j} y<b_{j} \wedge \bigwedge_{s} y \mid w_{s}
$$

where the as, bs, and ws are $\mathcal{Q}$-terms in $\bar{x}=\left(x_{1}, \ldots, x_{k}\right)$. Then the empty condition forces

$$
\forall \bar{x}, y, z\left(\gamma(\bar{x}, y) \wedge z>0 \rightarrow \bigvee_{0<r \in \mathbb{Q}} \exists y^{\prime}, y^{\prime \prime}\left(\gamma\left(\bar{x}, y^{\prime}\right) \wedge \gamma\left(\bar{x}, y^{\prime \prime}\right) \wedge r z=y^{\prime}-y^{\prime \prime}\right)\right)
$$

Proof. One need show merely that if $d_{1}, \ldots, d_{k}, e, f$ are distinct witnesses, $p$ is a condition extending

$$
\left\{a_{i}(\bar{d})<e: i \in I\right\} \cup\left\{e<b_{j}(\bar{d}): j \in J\right\} \cup\left\{e \mid w_{s}(\bar{d}): s \in S\right\} \cup\{f>0\},
$$

and $e^{\prime}$ and $e^{\prime \prime}$ are distinct witnesses not in $p$, then there is $r>0$ in $\mathbb{Q}$ for which

$$
\begin{aligned}
& p \cup\left\{a_{i}(\bar{d})<e^{\prime}: i \in I\right\} \cup\left\{e^{\prime}<b_{j}(\bar{d}): j \in J\right\} \cup\left\{e^{\prime} \mid w_{s}(\bar{d}): s \in S\right\} \\
& \cup\left\{a_{i}(\bar{d})<e^{\prime \prime}: i \in I\right\} \cup\left\{e^{\prime \prime}<b_{j}(\bar{d}): j \in J\right\} \cup\left\{e^{\prime \prime} \mid w_{s}(\bar{d}): s \in S\right\} \\
& \quad \cup\left\{r f=e^{\prime}-e^{\prime \prime}\right\}
\end{aligned}
$$

is a condition.

The condition $p$ may be satisfied in some $\mathbb{Q}^{n}$ by an assignment $\rho$. Since $f$ is positive in the simple divisible dimension group $\mathbb{Q}^{n}$, there is $r_{0}>0$ in $\mathbb{Q}$ such that for all rational $t \in\left[0, r_{0}\right), e \pm t f$ satisfies in $\mathbb{Q}^{n}$ all the inequalities in $\gamma(\bar{d}, y)$. Each incomparability $e \mid w_{s}$ is true in $\mathbb{Q}^{n}$ either because $e-w_{s}$ assumes both positive and negative values or because $e-w_{s}$ is always nonnegative (nonpositive) but not always zero and not always positive (negative). Call an incomparability of the second kind singular, and let $s_{1}, \ldots, s_{q}$ be the indices of all the singular incomparabilities. By copying $\rho q+1$ times and letting $e^{\prime}$ and $e^{\prime \prime}$ have the same denotation in $\mathbb{Q}^{n(q+1)}$ as $e$, one obtains an assignment $\sigma$ in $\mathbb{Q}^{n(q+1)}$ that satisfies $p$ and makes $e \pm t f=$ $e^{\prime} \pm t f=e^{\prime \prime} \pm t f$ satisfy all the inequalities in $\gamma(\bar{d}, y)$ whenever $t \in\left[0, r_{0}\right) \cap \mathbb{Q}$. One may now change $\sigma$ as follows to a new assignment $\tau$ in $\mathbb{Q}^{n(q+1)}$ that agrees with $\sigma$ on every witness but $e^{\prime}$. For $i=1, \ldots, q$ the incomparability $e \mid w_{s_{i}}$ is singular with respect to $\rho$, and so there is $j_{i}$ with $1 \leq j_{i} \leq n$ and

$$
\rho(e)_{j_{i}}=\rho\left(w_{s_{i}}\right)_{j_{i}} .
$$

Change $\sigma\left(e^{\prime}\right)$ to $\tau\left(e^{\prime}\right)$ by changing $\sigma\left(e^{\prime}\right)$ just at the coordinates $n i+j_{i}$ with $1 \leq i \leq q$ : $\sigma\left(e^{\prime}\right)_{n i+j_{i}}=\rho(e)_{j_{i}}$ becomes $\tau\left(e^{\prime}\right)_{n i+j_{i}}=\rho(e)_{j_{i}} \pm(1 / 2) r_{0} \rho(f)_{j_{i}}$, where the sign is - (+) just in case all coordinates of $\rho\left(e-w_{s_{i}}\right)$ are nonnegative (nonpositive). $\tau$ satisfies $p \cup\left\{\gamma\left(\bar{d}, e^{\prime}\right), \gamma\left(\bar{d}, e^{\prime \prime}\right)\right\}$, and every incomparability $e^{\prime} \mid w_{s}$ is nonsingular with 
respect to $\tau$ : i.e., $e^{\prime}-w_{s}$ assumes both positive and negative values under $\tau$. There is a positive rational $r_{1}<r_{0}$ such that for all rational $t \in\left[0, r_{1}\right)$, all $i=1, \ldots, n(q+1)$, and all $s$,

$$
\tau\left(e^{\prime}-w_{s}\right)_{i} \neq 0 \Rightarrow \tau\left(e^{\prime}-w_{s}\right)_{i} \text { and } \tau\left(e^{\prime}+t f-w_{s}\right)_{i} \text { have the same sign. }
$$

One concludes that $\tau$ satisfies

$$
\gamma\left(\bar{d}, e^{\prime}+(1 / 2) r_{1} f\right) \wedge(1 / 2) r_{1} f=\left(e^{\prime}+(1 / 2) r_{1} f\right)-e^{\prime} .
$$

If $\mu$ is the assignment in $\mathbb{Q}^{n(q+1)}$ with $\mu\left\lceil W \backslash\left\{e^{\prime}, e^{\prime \prime}\right\}=\tau \uparrow W \backslash\left\{e^{\prime}, e^{\prime \prime}\right\}, \mu\left(e^{\prime}\right)=\right.$ $\tau\left(e^{\prime}+(1 / 2) r_{1} f\right)$, and $\mu\left(e^{\prime \prime}\right)=\tau\left(e^{\prime}\right)$, then $\mu$ satisfies the second display of the first paragraph when $r=(1 / 2) r_{1}$.

Lemma 6.11 now implies that

Lemma 6.31. If $\gamma(\bar{x}, y)$ is a finite conjunction of inequalities, incomparabilities, and formulas $\exists z(\theta \wedge z \mid 0)$, where $\theta$ is a finite conjunction of inequalities in $\bar{x}=$ $\left(x_{1}, \ldots, x_{k}\right), y$, and the new variable $z$, then in any finitely generic dimension group,

$\forall \bar{x}, y, z\left(\gamma(\bar{x}, y) \wedge z>0 \rightarrow \exists g \in \mathbb{Q} \cdot z \exists y^{\prime}, y^{\prime \prime}\left(g>0 \wedge \gamma\left(\bar{x}, y^{\prime}\right) \wedge \gamma\left(\bar{x}, y^{\prime \prime}\right) \wedge g=y^{\prime}-y^{\prime \prime}\right)\right)$.

Proof. One may assume that $\gamma(\bar{x}, y)$ is

$$
\bigwedge_{i} a_{i}<y \wedge \bigwedge_{j} y<b_{j} \wedge \bigwedge_{s} y \mid w_{s} \wedge \bigwedge_{m} \exists z_{m}\left(\theta_{m} \wedge z_{m} \mid 0\right),
$$

where the $a \mathrm{~s}, b \mathrm{~s}$, and $w \mathrm{~s}$ are terms in $\bar{x}$, the $z \mathrm{~s}$ are distinct variables not among $\bar{x}, y$, and each $\theta_{m}$ is a conjunction of inequalities in $\bar{x}, y$, and $z_{m}$. If one applies Lemma 6.30 to

$$
\bigwedge_{i} a_{i}<y \wedge \bigwedge_{j} y<b_{j} \wedge \bigwedge_{s} y\left|w_{s} \wedge \bigwedge_{m} \theta_{m} \wedge z_{m}\right| 0
$$

invokes Lemma 6.11, and remembers that the theory of finitely generic dimension groups is complete, one reaches the desired conclusion.

This analogue of Lemma 6.9 allows one to establish Lemma 6.29 when $l=1$. Given $\zeta(\bar{x})=\mathrm{Q}_{1} y_{1} \gamma\left(\bar{x}, y_{1}\right)$ as in Lemma 6.29, one need treat only the case in which $\mathrm{Q}_{1}=\mathrm{Q}$. Since Q $y \gamma(\bar{x}, y)$ always implies $\exists y \gamma(\bar{x}, y)$, (i) and (ii) will be true if

(i) ${ }^{\prime}$ If $\gamma(\bar{x}, y)$ contains no identities, then $\exists y \gamma(\bar{x}, y)$ implies $\mathrm{Q} y \gamma(\bar{x}, y)$ in $\mathcal{M}$ and

(ii) $^{\prime}$ If $\mathrm{Q} y \gamma(\bar{x}, y)$ is satisfiable in $\mathcal{M}$, then $\exists y \gamma(\bar{x}, y)$ implies $\mathrm{Q} y \gamma(\bar{x}, y)$ in $\mathcal{M}$ are true. Fix $\bar{a} \in M^{k}$. If $\gamma$ contains no identities and $\mathcal{M} \models \exists y \gamma(\bar{a}, y)$, then Lemma 6.31 implies that the $\mathbb{Q}$-linear span of $\{b \in M: \mathcal{M} \models \gamma(\bar{a}, b)\}$ contains all positive elements of $M$; because the positive elements of $M$ generate the directed group $\mathcal{M}$, this linear span is $M$, and since $\mathcal{M}$ is an infinite-dimensional vector space (Lemma 6.23),$\{b \in M: \mathcal{M}=\gamma(\bar{a}, b)\}$ contains an infinite linearly independent set as desired. If $\mathrm{Q} y \gamma(\bar{x}, y)$ is satisfiable in $\mathcal{M}$, then no conjunct of $\gamma$ is an identity containing $y$ nontrivially; so the prenexing rule for $\mathrm{Q}$ and $\wedge$ allows one to assume that no conjunct of $\gamma$ is an identity, and the earlier argument yields the desired conclusion.

To handle the induction step, assume that $l>1$ and that Lemma 6.29 holds for formulas with $l-1$ initial quantifiers $\mathrm{Q}, \exists$. If $\gamma(\bar{x}, \bar{y})$ contains no identities, then the induction hypothesis implies that $\mathrm{Q}_{2} y_{2} \ldots \mathrm{Q}_{l} y_{l} \gamma(\bar{x}, \bar{y})$ is equivalent in $\mathcal{M}$ to 
$\exists y_{2} \ldots \exists y_{l} \gamma(\bar{x}, \bar{y})$, which by Lemma 3.3 is equivalent in $\mathcal{M}$ to a finite conjunction $\gamma^{\prime}\left(\bar{x}, y_{1}\right)$ of inequalities, incomparabilities, and formulas $\exists z(\theta \wedge z \mid 0)$ as above; the basis of the induction therefore shows that $\mathrm{Q}_{1} y_{1} \gamma^{\prime}\left(\bar{x}, y_{1}\right)$ is equivalent in $\mathcal{M}$ to $\exists y_{1} \gamma^{\prime}\left(\bar{x}, y_{1}\right)$, as desired. If $\zeta(\bar{x})=\mathrm{Q}_{1} y_{1} \ldots \mathrm{Q}_{l} y_{l} \gamma(\bar{x}, \bar{y})$ is satisfiable in $\mathcal{M}$, then $\mathrm{Q}_{2} y_{2} \ldots \mathrm{Q}_{l} y_{l} \gamma(\bar{x}, \bar{y})$ also is satisfiable in $\mathcal{M}$, and by the induction hypothesis is equivalent in $\mathcal{M}$ to $\exists y_{2} \ldots \exists y_{l} \gamma(\bar{x}, \bar{y})$. Lemma 3.3 supplies a finite conjunction $\gamma^{\prime \prime}\left(\bar{x}, y_{1}\right)$ of identities, inequalities, incomparabilities, and formulas $\exists z(\theta \wedge z \mid 0)$ that is equivalent in $\mathcal{M}$ to $\exists y_{2} \ldots \exists y_{l} \gamma(\bar{x}, \bar{y})$. The basis of the induction now implies that $\mathrm{Q}_{1} y_{1} \gamma^{\prime \prime}\left(\bar{x}, y_{1}\right)$ is equivalent in $\mathcal{M}$ either to $0 \neq 0$ or to $\exists y_{1} \gamma^{\prime \prime}\left(\bar{x}, y_{1}\right)$, and the induction step is complete.

Now one may verify the contrapositive of (vii). It has finitely many free variables $x_{1}, \ldots, x_{k}$ : so suppose $\bar{a} \in M^{k}$ and

$$
\mathcal{M} \models \neg \bigvee_{n \in \mathbb{N}} \mathrm{Q} v \beta_{n}(v, \bar{a}) .
$$

If each set $\left\{b \in M: \mathcal{M}=\beta_{n}(b, \bar{a})\right\}$ is contained in the linear span $V$ of $a_{1}, \ldots, a_{k}$, then $\left\{b \in M: \mathcal{M} \models \bigvee_{n \in \mathbb{N}} \beta_{n}(b, \bar{a})\right\}$ is contained in the finite-dimensional vector space $V$ and the desired conclusion follows; so assume $\mathcal{M} \models \beta_{n}(b, \bar{a})$. As in the verification of schema (vi), $\beta_{n}(v, \bar{x})$ is equivalent in $\mathcal{M}$ to a formula $\bigvee_{j=1}^{r} \beta_{j}^{\prime}(v, \bar{x})$, where each $\beta_{j}^{\prime}$ results from a finite conjunction of identities, inequalities, and incomparabilities when quantifiers $\mathrm{Q}$ and $\exists$ are applied to certain variables. Since the focus here is on $\left\{c \in M: \mathcal{M}=\bigvee_{j=1}^{r} \beta_{j}^{\prime}(c, \bar{a})\right\} \neq \emptyset$, one may assume that each formula $\beta_{j}^{\prime}(v, \bar{a})$ is satisfiable in $\mathcal{M}$. Lemma 6.29 implies that each formula $\beta_{j}^{\prime}(v, \bar{x})$ is equivalent in $\mathcal{M}$ to the existential quantification of a finite conjunction of identities, inequalities, and incomparabilities, and Lemma 3.3 makes each of these formulas equivalent in $\mathcal{M}$ to a finite conjunction $\gamma_{j}(v, \bar{x})$ of identities, inequalities, incomparabilities, and formulas $\exists z(\theta \wedge z \mid 0)$ as above. If no conjunct of $\gamma_{j}(v, \bar{x})$ is an identity with a nontrivial occurrence of $v$, then since $\gamma_{j}(v, \bar{a})$ is satisfiable in $\mathcal{M}$, Lemma 6.31 implies that the linear span of $\left\{c \in M: \mathcal{M} \models \gamma_{j}(c, \bar{a})\right\}$ contains all positive elements of $M$, and so all elements of the directed group $\mathcal{M}$; since $\mathcal{M}$ is an infinite-dimensional vector space (Lemma 6.23), $\left\{c \in M: \mathcal{M} \models \gamma_{j}(c, \bar{a})\right\} \subseteq\left\{c \in M: \mathcal{M} \models \beta_{n}(c, \bar{a})\right\}$ both contain an infinite linearly independent set, though $\mathcal{M}=\neg \mathrm{Q} v \beta_{n}(v, \bar{a})$. Thus each $\gamma_{j}(v, \bar{x})$ has a conjunct making $v$ a linear form in $\bar{x}$, and

$$
\left\{c \in M: \mathcal{M}=\beta_{n}(c, \bar{a})\right\}=\bigcup_{j=1}^{r}\left\{c \in M: \mathcal{M} \models \gamma_{j}(c, \bar{a})\right\} \subseteq V,
$$

as desired.

\section{Functorial existential Closure}

Corollaries 6.1 and 6.2 suggest that there is no natural candidate for the existential closure of the integers. Nevertheless, the present section will present several functors that embed nontrivial dimension groups in existentially closed ones.

Recall from Section 2 the diagonal embeddings $\mathcal{G} \rightarrow \widehat{\mathcal{G}}$ and $\mathcal{G} \rightarrow \mathcal{G}^{\aleph_{0}}$ of the divisible dimension group $\mathcal{G}$ viewed as a $\mathcal{Q}$-structure. If $\mathcal{M}$ is any nontrivial dimension group, one may define, for all ordinals $\alpha<\beta$, a $\mathcal{Q}$-structure $\mathcal{M}_{\alpha}$ and an embedding $\delta_{\alpha, \beta}: \mathcal{M}_{\alpha} \rightarrow \mathcal{M}_{\beta}$ recursively as follows. $\mathcal{M}_{0}$ is the divisible hull of $\mathcal{M}$, and when $\lambda$ is 0 or a limit ordinal and $i<\omega$,

$$
\mathcal{M}_{\lambda+2 i+1}=\mathcal{M}_{\lambda+2 i}^{\aleph_{0}} \text { and } \mathcal{M}_{\lambda+2 i+2}=\widehat{\mathcal{M}}_{\lambda+2 i+1} .
$$


If one pretends that the diagonal maps are inclusions, the $\delta_{\alpha, \beta}$ s will be inclusions and $\mathcal{M}_{\lambda}$ will be $\bigcup_{\alpha<\lambda} \mathcal{M}_{\alpha}$ when $\lambda$ is a limit ordinal; but the exact definitions are more complex. If $\lambda$ is 0 or a limit ordinal and $i<\omega, \delta_{\lambda+2 i, \lambda+2 i+1}: \mathcal{M}_{\lambda+2 i} \rightarrow \mathcal{M}_{\lambda+2 i}^{\aleph_{0}}$ and $\delta_{\lambda+2 i+1, \lambda+2 i+2}: \mathcal{M}_{\lambda+2 i+1} \rightarrow \widehat{\mathcal{M}}_{\lambda+2 i+1}$ are the corresponding diagonal maps. If $\alpha$ is an ordinal and $1<n<\omega$,

$$
\delta_{\alpha, \alpha+n}=\delta_{\alpha+n-1, \alpha+n} \circ \cdots \circ \delta_{\alpha+1, \alpha+2} \circ \delta_{\alpha, \alpha+1} .
$$

If $\lambda$ is a limit ordinal, $\alpha<\lambda$, and $1 \leq n<\omega$, then $\delta_{\alpha, \lambda+n}=\delta_{\lambda, \lambda+n} \circ \delta_{\alpha, \lambda}$. Finally, if $\lambda$ is a limit ordinal, $\left\{\mathcal{M}_{\alpha}\right\}_{\alpha<\lambda}$ and $\left\{\delta_{\alpha, \beta}\right\}_{\alpha<\beta<\lambda}$ are available, and

$$
\delta_{\beta, \gamma} \circ \delta_{\alpha, \beta}=\delta_{\alpha, \gamma} \text { whenever } \alpha<\beta<\gamma<\lambda,
$$

then $\mathcal{M}_{\lambda}$ is the direct limit of the system $\left(\left\{\mathcal{M}_{\alpha}\right\}_{\alpha<\lambda},\left\{\delta_{\alpha, \beta}\right\}_{\alpha<\beta<\lambda}\right)$ and $\delta_{\alpha, \lambda}$ is the induced homomorphism of $\mathcal{M}_{\alpha}$ with $\alpha<\lambda$ into $\mathcal{M}_{\lambda}$.

Every $\mathcal{M}_{\alpha}$ is a strictly directed divisible dimension group. The argument for Theorem 2.3 shows that

Lemma 7.1. If $\mathcal{M}$ is a nontrivial divisible dimension group satisfying the antecedent of an instance of schema (2) or (3), then $\widehat{\mathcal{M}}$ satisfies the image under the diagonal embedding $\mathcal{M} \rightarrow \widehat{\mathcal{M}}$ of the consequent of the schema.

Also, the argument for Theorem 2.6 shows that

Lemma 7.2. If $\mathcal{M}$ is a nontrivial divisible dimension group satisfying the antecedent of an instance of schema (4), (5), or (6), then $\mathcal{M}^{\aleph_{0}}$ satisfies the image under the diagonal embedding $\mathcal{M} \rightarrow \mathcal{M}^{\aleph_{0}}$ of the consequent of the schema.

Thus

Theorem 7.3. If $\mathcal{M}$ is a nontrivial dimension group and $\lambda$ is a limit ordinal, then $\mathcal{M}_{\lambda}$ is an e.c. dimension group into which $\mathcal{M}$ is embedded by $\delta_{0, \lambda} \uparrow M$.

Modulo the theory of dimension groups, schema (3) implies that

$$
\forall y>0(x+y>0) \leftrightarrow x \geq 0:
$$

that is, there are no pseudo-positive elements (4], p. 7). If $0<a$ in $\mathcal{G}$, then $(0, a, 0, a, \ldots) \in G^{\aleph_{0}}$ obeys the left-hand side but not the right; if $\mathcal{G}$ is not linearly ordered, then one may find a similar example in $\widehat{G}$. One may exploit these observations to show that when $\mathcal{M}$ is a nontrivial dimension group, $\mathcal{M}_{\alpha+1}$ is not existentially closed.

The proofs of Theorems 2.3 and 2.6 rely on elements of $\mathcal{G}^{\aleph_{0}}$ or $\widehat{\mathcal{G}}$ with finite range to verify consequents of schemas whose antecedents are true in $\mathcal{G}$. The elements from $\mathcal{G}^{\aleph_{0}}$ or $\widehat{\mathcal{G}}$ with finite range form substructures $\left(\mathcal{G}^{\aleph_{0}}\right)_{f} \subseteq \mathcal{G}^{\aleph_{0}}$ and $(\widehat{\mathcal{G}})_{f} \subseteq \widehat{\mathcal{G}}$, and the diagonal maps still embed $\mathcal{G}$ in $\left(\mathcal{G}^{\aleph_{0}}\right)_{f}$ and in $(\widehat{\mathcal{G}})_{f}$. If in the previous definitions one replaces the operations $\mathcal{G} \mapsto \mathcal{G}^{\aleph_{0}}$ and $\mathcal{G} \mapsto \widehat{\mathcal{G}}$ by $\mathcal{G} \mapsto\left(\mathcal{G}^{\aleph_{0}}\right)_{f}$ and $\mathcal{G} \mapsto(\widehat{\mathcal{G}})_{f}$ one obtains structures $\left(\mathcal{M}_{\alpha}\right)_{f}$, and the earlier arguments show that $\delta_{0, \lambda} \uparrow M$ embeds $\mathcal{M}$ in the e.c. dimension group $\left(\mathcal{M}_{\lambda}\right)_{f}$ when $\lambda$ is a limit ordinal. Yet the restriction to elements with finite range makes every positive element of $\left(M_{\alpha+1}\right)_{f}$ bounded above by positive elements of $\left(M_{\alpha}\right)_{f}$, and so

Corollary 7.4. If $\mathcal{M}$ is a nontrivial dimension group and $\lambda$ is a limit ordinal, then $\left(\mathcal{M}_{\lambda}\right)_{f}$ is an e.c. dimension group, into which $\mathcal{M}$ is embedded by $\delta_{0, \lambda} \uparrow M$, whose positive elements are bounded above by elements of $\delta_{0, \lambda}(M)$. 
Nontrivial dimension groups, and embeddings between such groups, form a category $\mathcal{C}$, and one may show that the mappings $\mathcal{M} \mapsto \mathcal{M}_{\lambda}$ and $\mathcal{M} \mapsto\left(\mathcal{M}_{\lambda}\right)_{f}$ expand to functors from $\mathcal{C}$ into $\mathcal{C}$. Since any embedding $\theta: \mathcal{M} \rightarrow \mathcal{N}$ between dimension groups extends uniquely to an embedding $\theta_{0}: \mathcal{M}_{0} \rightarrow \mathcal{N}_{0}$ between their divisible hulls, one may reach the desired conclusion by showing that if $\theta$ is an embedding between divisible dimension groups $\mathcal{M}$ and $\mathcal{N}$, then $\mathcal{M}^{\aleph_{0}}\left(\left(\mathcal{M}^{\aleph_{0}}\right)_{f}, \widehat{\mathcal{M}},(\widehat{\mathcal{M}})_{f}\right)$ may be embedded into $\mathcal{N}^{\aleph_{0}}\left(\left(\mathcal{N}^{\aleph_{0}}\right)_{f}, \widehat{\mathcal{N}},(\widehat{\mathcal{N}})_{f}\right)$ in an appropriate fashion. Because the same arguments apply to $\mathcal{M}^{\aleph_{0}}, \widehat{\mathcal{M}}$ and to $\left(\mathcal{M}^{\aleph_{0}}\right)_{f},(\widehat{\mathcal{M}})_{f}$, one may concentrate on $\mathcal{M}^{\aleph_{0}}$ and $\widehat{\mathcal{M}}$.

If $\delta_{\mathcal{M}}: \mathcal{M} \rightarrow \mathcal{M}^{\aleph_{0}}$ is the diagonal embedding, then one wants to associate, with any embedding $\theta: \mathcal{M} \rightarrow \mathcal{N}$ in $\mathcal{C}$, an embedding $\theta^{\aleph_{0}}: \mathcal{M}^{\aleph_{0}} \rightarrow \mathcal{N}^{\aleph_{0}}$ for which

$$
\theta^{\aleph_{0}} \circ \delta_{\mathcal{M}}=\delta_{\mathcal{N}} \circ \theta
$$

and

$$
(\gamma \circ \theta)^{\aleph_{0}}=\gamma^{\aleph_{0}} \circ \theta^{\aleph_{0}}
$$

whenever $\gamma: \mathcal{N} \rightarrow \mathcal{P}$ is an embedding in $\mathcal{C}$. Clearly the map $\theta^{\aleph_{0}}: \prod_{i \in \mathbb{N}} M \rightarrow$ $\prod_{i \in \mathbb{N}} N$ given for $a \in \prod_{i \in \mathbb{N}} M$ by

$$
\theta^{\aleph_{0}}(a)=\theta \circ a
$$

does the job.

If $\widehat{\delta}_{\mathcal{M}}: \mathcal{M} \rightarrow \widehat{\mathcal{M}}$ is the diagonal embedding, then one wants to associate, with any embedding $\theta: \mathcal{M} \rightarrow \mathcal{N}$ between divisible groups in $\mathcal{C}$, an embedding $\widehat{\theta}: \widehat{\mathcal{M}} \rightarrow$ $\widehat{\mathcal{N}}$ for which

$$
\widehat{\theta} \circ \widehat{\delta}_{\mathcal{M}}=\widehat{\delta}_{\mathcal{N}} \circ \theta
$$

and

$$
\widehat{\gamma \circ \theta}=\widehat{\gamma} \circ \widehat{\theta}
$$

whenever $\gamma: \mathcal{N} \rightarrow \mathcal{P}$ is an embedding between divisible groups in $\mathcal{C}$. If $\prec \in \mathcal{O}(\mathcal{N})$ let

$$
\prec_{\theta}=\left\{(a, b) \in M^{2}: \theta(a) \prec \theta(b)\right\} .
$$

Because $\prec_{\theta} \in \mathcal{O}(\mathcal{M})$, one may define $\widehat{\theta}: \widehat{\mathcal{M}} \rightarrow \widehat{\mathcal{N}}$ for $a \in \widehat{\mathcal{M}}$ by

$$
\widehat{\theta}(a)(\prec)=(\theta \circ a)\left(\prec_{\theta}\right) \text { whenever } \prec \in \mathcal{O}(\mathcal{N}) .
$$

One easily shows that $\widehat{\theta}$ is a homomorphism of $\widehat{\mathcal{M}}$ into $\widehat{\mathcal{N}}$ and that $\widehat{\gamma \circ \theta}=\widehat{\gamma} \circ \widehat{\theta}$ whenever $\gamma: \mathcal{N} \rightarrow \mathcal{P}$ is an embedding in $\mathcal{C}$. $\widehat{\theta}$ will be an embedding just in case

Lemma 7.5. If $\theta: \mathcal{M} \rightarrow \mathcal{N}$ is an embedding between divisible groups in $\mathcal{C}$, then $\mathcal{O}(\mathcal{M})=\left\{\prec_{\theta}: \prec \in \mathcal{O}(\mathcal{N})\right\}$

Proof. One need show merely that any $\sqsubset$ in $\mathcal{O}(\mathcal{M})$ is $\prec_{\theta}$ for some $\prec \in \mathcal{O}(\mathcal{N})$. Given $\sqsubset \in \mathcal{O}(\mathcal{M})$, let

$$
S=\{p+\theta(m): p \geq 0 \text { in } \mathcal{N} \text { and } m \sqsupseteq 0 \text { in } \mathcal{M}\} .
$$

$S$ contains the nonnegative elements of $N$ and is closed under addition and nonnegative rational multiplication. If $S \cap(-S)=\{0\}$ the proof of Lemma 2.2 will show that $S$ is contained in the nonnegative cone of some $\prec \in \mathcal{O}(\mathcal{N})$, and $\sqsubset=\prec_{\theta}$. So, assume

$$
q=p+\theta(m)=-\left(p^{\prime}+\theta\left(m^{\prime}\right)\right),
$$


where $p, p^{\prime} \geq 0$ in $\mathcal{N}$ and $m, m^{\prime} \sqsupseteq 0$ in $\mathcal{M}$. $0 \leq p+p^{\prime}=-\theta\left(m+m^{\prime}\right)$. If $p+p^{\prime}>0$, then $\theta\left(m+m^{\prime}\right)=-\left(p+p^{\prime}\right)<0$, and since $\theta$ is an embedding, $m+m^{\prime}<0$ and $m+m^{\prime} \sqsubset 0$. Thus $p+p^{\prime}=0$, and since $p, p^{\prime} \geq 0, p=p^{\prime}=0, \theta\left(m+m^{\prime}\right)=$ $-\left(p+p^{\prime}\right)=0, m+m^{\prime}=0$ because $\theta$ is an embedding, and $m=0=m^{\prime}$ since $m, m^{\prime} \sqsupseteq 0$. So $S \cap(-S)=\{0\}$, as desired.

One obtains an interesting variant of these functors if one replaces the operation $\mathcal{M} \mapsto \mathcal{M}^{\aleph_{0}}$ by $\mathcal{M} \mapsto \mathcal{M}^{\mathcal{F}}$, where $\mathcal{F}$ is the cofinite filter on $\mathbb{N}$ and

$$
\mathcal{M}^{\mathcal{F}}=\mathcal{M}^{\aleph_{0}} / \mathcal{F}
$$

is the corresponding reduced product. The diagonal map $\delta: \mathcal{M} \rightarrow \mathcal{M}^{\aleph_{0}}$ induces an embedding $\delta^{\mathcal{F}}: \mathcal{M} \rightarrow \mathcal{M}^{\mathcal{F}}$ given by

$$
\delta^{\mathcal{F}}(m)=[\delta(m)],
$$

where $[a]$ is the class of $a \in M^{\aleph_{0}}$ in $\mathcal{M}^{\aleph_{0}} / \mathcal{F}$. Because the elements of $M^{\aleph_{0}}$ used in the proofs of Theorem 2.6 and Lemma 7.2 are periodic, the earlier arguments show that

Lemma 7.6. If $\mathcal{M}$ is a nontrivial divisible dimension group satisfying the antecedent of an instance of schema (4), (5), or (6), then $\mathcal{M}^{\mathcal{F}}$ satisfies the image under the diagonal embedding $\mathcal{M} \rightarrow \mathcal{M}^{\mathcal{F}}$ of the consequent of the schema.

Replace, in the definition of $\mathcal{M}_{\alpha}$, each $\mathcal{G}^{\aleph_{0}}$ by $\mathcal{G}^{\mathcal{F}}$ and each corresponding $\delta$ by $\delta^{\mathcal{F}}$. One obtains dimension groups $\left(\mathcal{M}_{\alpha}\right)_{\mathcal{F}}$ and embeddings $\delta_{\alpha, \beta}^{\mathcal{F}}:\left(\mathcal{M}_{\alpha}\right)_{\mathcal{F}} \rightarrow\left(\mathcal{M}_{\beta}\right)_{\mathcal{F}}$ for all ordinals $\alpha<\beta$, and

Theorem 7.7. If $\mathcal{M}$ is a nontrivial dimension group and $\lambda$ is a limit ordinal, then $\left(\mathcal{M}_{\lambda}\right)_{\mathcal{F}}$ is an e.c. dimension group into which $\mathcal{M}$ is embedded by $\delta_{0, \lambda}^{\mathcal{F}} \uparrow M$. If $\lambda$ has uncountable cofinality, then $\left(\mathcal{M}_{\lambda}\right)_{\mathcal{F}}$ is $\omega_{1}$-existentially saturated.

Proof. The key to the second claim is that $\mathcal{G}^{\mathcal{F}}$ is always $\omega_{1}$-saturated ([6]). Suppose $A \subseteq\left(M_{\lambda}\right)_{\mathcal{F}}$ is at most countable and $\left\{\varphi_{i}\left(x_{1}, \ldots, x_{k}\right): i \in \mathbb{N}\right\}$ is a collection of existential $\mathcal{Q}_{A}$-formulas finitely satisfiable in $\left(\mathcal{M}_{\lambda}\right)_{\mathcal{F}}$. Since $\lambda$ has uncountable cofinality there are a limit ordinal $\theta<\lambda$ and $j<\omega$ such that the elements of $A$ come from $\left(M_{\theta+2 j+1}\right)_{\mathcal{F}}$ and $\left\{\varphi_{i}(\bar{x}): i \in \mathbb{N}\right\}$ is finitely satisfiable in $\left(\mathcal{M}_{\theta+2 j+1}\right)_{\mathcal{F}} 16$ Because this structure is $\omega_{1}$-saturated, $\left\{\varphi_{i}(\bar{x}): i \in \mathbb{N}\right\}$ is satisfiable in $\left(\mathcal{M}_{\theta+2 j+1}\right)_{\mathcal{F}}$; because the $\varphi$ s are existential, the desired conclusion follows.

Every embedding $\theta: \mathcal{M} \rightarrow \mathcal{N}$ in $\mathcal{C}$ yields an embedding $\theta^{\mathcal{F}}: \mathcal{M}^{\mathcal{F}} \rightarrow \mathcal{N}^{\mathcal{F}}$ with

$$
\theta^{\mathcal{F}}([a])=[\theta \circ a]
$$

for all $a \in M^{\aleph_{0}}$ and

$$
(\gamma \circ \theta)^{\mathcal{F}}=\gamma^{\mathcal{F}} \circ \theta^{\mathcal{F}}
$$

whenever $\gamma: \mathcal{N} \rightarrow \mathcal{P}$ is an embedding in $\mathcal{C}$; so as before $\mathcal{M} \mapsto\left(\mathcal{M}_{\lambda}\right)_{\mathcal{F}}$ yields a functor.

Though the functorial embeddings $\mathcal{M} \rightarrow \mathcal{M}_{\lambda}, \mathcal{M} \rightarrow\left(\mathcal{M}_{\lambda}\right)_{f}$, and $\mathcal{M} \rightarrow\left(\mathcal{M}_{\lambda}\right)_{\mathcal{F}}$ provide a contrast to Corollaries 6.1 and 6.2, these functors do not have all the properties one might wish for a closure operation. By incorporating operations such as $\mathcal{G} \mapsto \mathcal{G}^{\aleph_{0}}$ or $\mathcal{G} \mapsto \widehat{\mathcal{G}}$, these functors always produce proper extensions of the nontrivial dimension group with which one starts, and so cannot be idempotent or

\footnotetext{
${ }^{16}$ More accurately: $A \subseteq \operatorname{ran} \delta_{\theta+2 j+1, \lambda}^{\mathcal{F}}$ and the images under $\left(\delta_{\theta+2 j+1, \lambda}^{\mathcal{F}}\right)^{-1}$ of the $\varphi$ s are finitely satisfiable in $\left(\mathcal{M}_{\theta+2 j+1}\right) \mathcal{F}$.
} 
fix dimension groups that already are existentially closed. For certain applicationsfor example, to the countable dimension groups arising from $\mathrm{AF} C^{*}$-algebras - one might want to pick a countable e.c. extension of a given countable dimension group, but the operations already considered will not have this property. Yet a different approach will produce, for certain classes of nontrivial dimension groups, functorial embeddings into e.c. dimension groups that are idempotent and do not increase cardinality.

If $\kappa$ is an infinite cardinal, then there is a set of dimension groups that contains an isomorphic copy of every dimension group of cardinality at most $\kappa$. Because the class of dimension groups is inductive and has the amalgamation property (Theorem 4.1), there is a dimension group $\mathcal{Z}$ into which all dimension groups of cardinality at most $\kappa$ embed, and since every dimension group has an e.c. extension one may assume that $\mathcal{Z}$ is e.c.17 Each nonuniversal axiom of $T_{1, \ldots, 6}$ is $\forall \exists$ in $\mathcal{Q}_{\omega_{1} \omega}$, and so the axiom of choice provides corresponding Skolem functions in $\mathcal{Z}$ : for example, a Skolem function $f$ corresponding to an instance of schema (3) would obey

$$
\bigwedge_{\bar{m} \in \mathbb{N}^{n} \backslash\{\overline{0}\}} 0 \not \leq \sum_{i} m_{i} a_{i} \rightarrow \bigwedge_{i} a_{i}<f(\bar{a}) \wedge 0 \nless f(\bar{a}),
$$

while a Skolem function $g$ corresponding to an instance of schema (4) would obey

$$
\begin{aligned}
\bigwedge_{s} \bigwedge_{i} a_{i}<y_{s} & \wedge \bigwedge_{j} y_{s}<b_{j} \wedge y_{s} \mid w_{s} \\
& \rightarrow \bigwedge_{i} a_{i}<g(\bar{a}, \bar{b}, \bar{y}, \bar{w}) \wedge \bigwedge_{j} g(\bar{a}, \bar{b}, \bar{y}, \bar{w})<b_{j} \wedge \bigwedge_{s} g(\bar{a}, \bar{b}, \bar{y}, \bar{w}) \mid w_{s} .
\end{aligned}
$$

If $\mathcal{M} \subseteq \mathcal{Z}$ is nontrivial, the closure $c(\mathcal{M})$ of $\mathcal{M}$ in $\mathcal{Z}$ under rational multiplication and these countably many Skolem functions is an e.c. dimension group, of the same cardinality as $\mathcal{M}$, with $\mathcal{M} \subseteq c(\mathcal{M}) \subseteq \mathcal{Z}$ as $\mathcal{L}^{<}$-structures, and the idempotent operation $c$ preserves inclusions and unions of chains. The category $\mathcal{C}_{1}$ of nontrivial substructures of $\mathcal{Z}$, with inclusions as maps, thus admits an idempotent functor into itself that embeds such substructures into e.c. substructures of the same cardinality, and $\mathcal{C}_{1}$ includes isomorphic copies of all dimension groups of cardinality at most $\kappa$.

A similar argument applies to dimension groups with distinguished order unit. One exploits the corresponding amalgamation property (Corollary 4.5) and the corresponding embedding theorem (Corollary 4.3) to build an e.c. dimension group $\mathcal{Z}^{\prime}$, with distinguished order unit $u$, into which all nontrivial dimension groups with distinguished order unit and of cardinality at most $\kappa$ admit $\mathcal{L}^{<} \cup\{u\}$-embeddings. Using Skolem functions as before for divisibility and the nonuniversal axioms of $T_{1, \ldots, 6}$, one obtains a category $\mathcal{C}_{2}$ of $\mathcal{L}^{<} \cup\{u\}$-substructures of $\mathcal{Z}^{\prime}$, with inclusions as maps, that admits an idempotent functor into itself that embeds substructures into existentially closed ones of the same cardinality.

Note finally that if one abandons the goal of functorial embedding one may modify the operations $\mathcal{G} \rightarrow \mathcal{G}^{\aleph_{0}}$ and $\mathcal{G} \rightarrow \widehat{\mathcal{G}}$ to embed a countable dimension group $\mathcal{M}$ in a countable e.c. dimension group. Without abandoning functorial embedding one may replace the clause

$$
\mathcal{M}_{2 i+1}=\mathcal{M}_{2 i}^{\aleph_{0}}
$$

by

$$
\mathcal{M}_{2 i+1}^{\prime}=\mathcal{M}_{2 i}^{\prime} \times \cdots \times \mathcal{M}_{2 i}^{\prime}
$$

\footnotetext{
${ }^{17}$ If $\kappa=\aleph_{0}$ an easy argument shows that one may let $\mathcal{Z}=\left(\mathbb{Z}_{\omega_{1}}\right)_{\mathcal{F}}$.
} 
where the product has $i+1$ factors. But functorial embedding seems to disappear when one replaces

$$
\mathcal{M}_{2 i+2}=\widehat{\mathcal{M}}_{2 i+1}
$$

by

$$
\mathcal{M}_{2 i+2}^{\prime}=\mathcal{M}_{2 i+1}^{\prime} \times\left(M_{2 i+1}^{\prime},+,-, 0, \prec\right)
$$

where $\prec \in \mathcal{O}\left(\mathcal{M}_{2 i+1}^{\prime}\right)$ is chosen to make $a_{1}, \ldots, a_{n} \prec 0$ for some $a_{1}, \ldots, a_{n} \in M_{2 i+1}^{\prime}$ with $\bigwedge_{\bar{m} \in \mathbb{N}^{n} \backslash\{\overline{0}\}} 0 \not \leq \sum_{i} m_{i} a_{i}$. As long as every tuple of elements, with this last property, that arises at any stage is handled at some later stage, one produces an e.c. dimension group when the direct limit over all finite stages is taken, and if $\mathcal{M}$ is countable one can arrange that all tuples are handled in this way.

\section{Some open PRoblems}

Any Skolem functions for $\mathcal{Z}\left(\mathcal{Z}^{\prime}\right)$ produce a corresponding idempotent functor embedding nontrivial dimension groups (with distinguished order unit) in e.c. groups of the same kind and cardinality. May one obtain functors with extra desirable properties by starting with Skolem functions having special properties?

Many of the properties discovered in Section 6 reveal ways in which finitely generic dimension groups are small, but there seems no reason to suppose that these properties characterize finitely generic dimension groups among e.c. dimension groups 18 Though Theorem 6.28 shows that finitely generic dimension groups may not be small in the sense of lacking proper elementary substructures, is there some other minimality condition that distinguishes finitely generic dimension groups among the e.c. dimension groups?

A nontrivial simple dimension group contained in no simple e.c. dimension group appears in Section 4, but it does not characterize $\mathcal{L} \cup\{u\}$-structures e.c. within the class $\mathcal{S}$ of nontrivial simple dimension groups with distinguished order unit. One can show that the groups e.c. in $\mathcal{S}$ are divisible, and when viewed as $\mathcal{Q}$-structures are exactly the models of

$$
T_{1,2,4,5,6} \cup\left\{u>0, \forall y>0 \forall x \bigvee_{n \geq 1} x \leq n y\right\}
$$

that satisfy the schema

$$
\left(\bigvee_{0<r \in \mathbb{Q}} \bigwedge_{\bar{m} \in \mathbb{N}^{n} \backslash\{\overline{0}\}} 0 \not \leq \sum_{i} m_{i}\left(a_{i}+r u\right)\right) \leftrightarrow \exists y\left(\bigwedge_{i} a_{i}<y \wedge 0 \nless y\right)
$$

In this new context one still may prove an analogue of Theorem 3.5. but what about analogues of other results?

Finally, Section 4 proves and exploits results about ideals in e.c. dimension groups but never takes quotients. Under what circumstances is the quotient of an e.c. dimension group by an ideal again e.c.?

\footnotetext{
${ }^{18}$ Added in proof: After submitting this paper, the author found that the only automorphisms of finitely generic dimension groups are multiplications by positive rationals, and that while no finitely generic dimension group has an arithmetic diagram, there are e.c. dimension groups, with recursive diagram, that have this smallest possible automorphism group and enjoy all the special properties established in Section 6 for countable finitely generic dimension groups (Theorem 6.13 Corollary 6.16 Theorem 6.18 and Corollary 6.26).
} 


\section{REFERENCES}

[1] I. Ben Yaacov, A. Berenstein, C. W. Henson, and A. Usvyatsov, Model theory for metric structures. In Z. Chatzidakis, D. Macpherson, A. Pillay, and A. Wilkie, eds., Model Theory with Applications to Algebra and Analysis, Volume 2, London Math. Soc. Lecture Note Ser. No. 350, Cambridge University Press, Cambridge, 2008, pp. 315-427. MR2436146 (2009j:03061)

[2] K. B. Bruce, Model-theoretic forcing in logic with a generalized quantifier, Ann. Math. Logic 13 (1978), 225-265. MR0491860 (80c:03033)

[3] G. Elliott, On the classification of inductive limits of sequences of semisimple finitedimensional algebras, J. Algebra 38 (1976), 29-44. MR0397420 (53:1279)

[4] L. Fuchs, Riesz vector spaces and Riesz algebras, Queen's Papers in Pure and Appl. Math. No. 1, Queen's University, Kingston, Ontario, 1966. MR0203436 (34:3288)

[5] W. Hodges, Building models by games, London Math. Soc. Stud. Texts No. 2, Cambridge University Press, Cambridge, 1985. MR0812274 (87h:03045)

[6] B. Jónsson and P. Olin, Almost direct products and saturation, Compos. Math. 20 (1968), 125-132. MR 0227004 (37:2589)

[7] H. J. Keisler, Logic with the quantifier "there exist uncountably many," Ann. Math. Logic 1 (1970), 1-93. MR0263616 (41:8217)

[8] P. Scowcroft, A representation of convex semilinear sets, Algebra Universalis 62 (2009), 289327. MR 2661381 (2011h:52007)

[9] P. Scowcroft, Some model-theoretic correspondences between dimension groups and AF algebras, Ann. Pure Appl. Logic 162 (2011), 755-785.

Department of Mathematics and Computer Science, Wesleyan University, MiddleTOWn, Connecticut 06459

E-mail address: pscowcroft@wesleyan.edu 